Laboratory study of the wave-induced mean flow and set-down in unidirectional surface gravity wavepackets on finite water depth

Calvert, $\mathrm{R}$

http://hdl.handle.net/10026.1/14843

10.1103/PhysRevFluids.4.114801

Physical Review Fluids

American Physical Society

All content in PEARL is protected by copyright law. Author manuscripts are made available in accordance with publisher policies. Please cite only the published version using the details provided on the item record or document. In the absence of an open licence (e.g. Creative Commons), permissions for further reuse of content should be sought from the publisher or author. 


\title{
laboratory study of the wave-induced mean flow and set-down in unidirectional surface gravity wavepackets on finite water depth
}

\author{
R. Calvert, ${ }^{1,2}$ C. Whittaker, ${ }^{3}$ A. Raby, ${ }^{4}$ P.H. Taylor, ${ }^{5}$ A.G.L. Borthwick, ${ }^{2}$ and T.S. van den Bremer ${ }^{1}$ \\ ${ }^{1}$ Department of Engineering Science, University of Oxford, Oxford OX1 3PJ, UK \\ ${ }^{2}$ School of Engineering, University of Edinburgh, Edinburgh EH9 3FB, UK \\ ${ }^{3}$ Department of Civil and Environmental Engineering, \\ University of Auckland, Auckland 1010, New Zealand \\ ${ }^{4}$ School of Engineering, University of Plymouth, Plymouth PL4 8AA, UK \\ ${ }^{5}$ Faculty of Engineering and Mathematical Sciences, \\ University of Western Australia, Crawley WA 6009, Australia
}

\begin{abstract}
The net movement of Lagrangian particles under water waves comprises a Stokes drift in the direction of wave propagation and an Eulerian return flow in the opposing direction. Accurate prediction of the Eulerian return flow in the ocean is of importance in modelling the transport of plastic pollution, oil, wreckage, and sediment. Herein, we derive a multiple-scales solution for the Eulerian mean flow under wavepackets that is valid for all water depths, both relative to the length of the wave and the length of the wavepacket. To validate this solution, we carry out Particle Tracking Velocimetry experiments in a long flume to extract the mean motion from Lagrangian seeding particles under wavepackets, finding good agreement. The extraction technique is able to deal with small background motion and sub-harmonic error waves associated with wave generation by the paddle, the latter being relatively large in finite-depth flume experiments. In finite depth, the return flow is forced by both the divergence of the Stokes transport on the wavepacket scale and the formation of a non-negligible mean set-down underneath the packet, which acts like a bounding streamtube in the form of a convergent-divergent duct. The magnitude of the horizontal return flow is thus enhanced, with particular relevance to transport in the finite-depth coastal environment.
\end{abstract}

Keywords: Wave-induced transport, Stokes drift, Lagrangian displacement, wavepackets, set-down, finite depth.

\section{INTRODUCTION}

Although fluid particles under progressive, small-amplitude surface gravity waves predominantly move forwards and backwards with the linear motion, they undergo a small forward net transport known as the Stokes drift [1]. Realistic seas are not regular waves but are composed of wavepackets, which can be represented as a linear superposition of waves of different frequencies [2]. In the limit of a narrow-banded spectrum, these packets can also be obtained by the amplitude modulation of a single frequency wave. Given that Stokes drift depends on the square of the local wave amplitude, its associated mass transport becomes divergent at packet-scale: mass transport is larger at the centre of the group than at leading and trailing edges. This divergence must be balanced by a return flow in the direction opposite to wave propagation, which also causes a depression in the wave-averaged free surface under the wavepacket, known as the set-down [3]. The depression in the wave-averaged free surface can be thought of as further enhancing the magnitude of the return flow.

Together, Stokes drift and the wave-induced Eulerian return flow make up the wave-induced Lagrangian velocity field, and both occur at second-order in a Stokes expansion. The Lagrangian velocity field is important in the prediction of species transport in the ocean, encompassing the movement of sediment, plastic, oil, and wreckage (see van den Bremer \& Breivik [4] for a recent review of Stokes drift and its applications). Because of its potentially large damage to marine wildlife, plastic, and more specifically microplastic, has recently attracted a great deal of attention $[5,6]$. Although recent research has focused on documenting the concentrations of plastic in the coastal region [7], and predicting locations of plastic accumulation [8], the effect of wave-induced plastic transport is yet to be fully explored. Wave-induced transport must also be included in the search for wreckage, such as concerning the 2014 MH370 airplane crash in the Indian Ocean [9]. Stokes drift is also important in the modelling of oil spills [10-12] and in sediment transport $[13,14]$.

Although it can be ignored in sufficiently deep water [15], the set-down of the wave-averaged free surface becomes important when the water depth is finite. Ignoring set-down or incorrectly reproducing it in numerical or laboratory experiments has been shown to lead to incorrect predictions of run-up on beaches [16]. Generation of wavepackets using linearly controlled wave paddles leads to spurious 'error waves' on the scale of the group, which travel ahead 
of the wavepacket as free waves, typically at the shallow-water phase velocity in laboratory flumes [17] (see also the discussion in $[16,18]$ ). Second-order wave generation, where the sub-harmonic frequencies represent the correct generation of the mean flow and the set-down of the wave-averaged free surface, has been proposed to remedy this $[19,20]$. Implementation of second-order wave generation can however be limited by the requirement of a large paddle stroke on the scale of the group [17].

Historically, several approaches have been taken to derive analytical solutions for the mean flow and the waveaveraged free surface under wavepackets. These solutions can be classified on the basis of two scales: the water depth $d$ relative to carrier wavelength, namely $k_{0} d$ with $k_{0}$ denoting the carrier wavenumber, and the water depth relative to packet length, namely $d / \sigma$ with $\sigma$ denoting the packet length scale. Table I shows the regimes based on these two nondimensional numbers for which solutions have been proposed in the literature. Assuming the return flow is shallow, i.e. $d / \sigma \ll 1$, such that the horizontal return flow is uniform over depth, Longuet-Higgins and Stewart [3], Davey and Stewartson [21], Brinch-Nielsen and Jonsson [22] and Mei et al. [23] presented solutions for the set-down and return flow. These solutions can describe water depths that are shallow, deep or intermediate with respect to the carrier wave length $\left(k_{0} d \ll 1, k_{0} d \gg 1\right.$ or $k_{0} d=\mathcal{O}(1)$, respectively). When $d / \sigma \gg 1$, the return flow does not extend to the bottom of the ocean floor, thus is considered a deep return flow and has been modelled by Dysthe [24] (and van den Bremer and Taylor [25]). None of these multiple-scales solutions are valid for an intermediate depth return flow, $d / \sigma=\mathcal{O}(1)$. Taking a different approach, Dalzell [26] represents the wavepacket as an infinite sum over wavenumber, which leads to a solution for the set-down and mean flow in the form of 'frequency-difference' terms in a double summation over wavenumber. Dalzell [26] makes no assumptions concerning the depth of the return flow or carrier wave, but the solutions do not enable simple evaluation of Lagrangian particle displacements. Expressions for these 'frequency-difference' terms can also be distilled from [3, 27-30] (see [31] for a recent re-derivation that also includes pressure).

Experimentally, many authors have considered Stokes drift in laboratory wave flumes. There remains some confusion in the literature whether a net drift should be observed (see the discussion in [4,32-34]). In addition to the solutions to the irrotational water wave equations [35,36], streaming in the boundary layers [33,37,38], convection of vorticity from the ends of the tank into the interior of the fluid [37,39] (or conduction from the free surface and bottom boundary layers [40]) or enhanced transport for particles on the surface in breaking waves may play a role [34, 41, 42]. Recently, van den Bremer et al. [43] have demonstrated experimentally that Lagrangian transport by the combination of Stokes drift and the Eulerian return flow underneath uni-directional, deep-water surface gravity wavepackets is in good agreement with leading-order solutions to the irrotational water wave equations.

This paper derives solutions for the mean flow and the wave-averaged free surface using the multiple-scales method, which are valid in arbitrary depths relative to the scale of the packet and the Eulerian return flow. The theory recovers all regimes for which solutions have previously been derived (see table I), and the predicted set-down matches the multi-component theory of Dalzell [26] in the narrow-banded limit. In doing so, we correct incomplete predictions in the finite-depth regime by [44]. We calculate Lagrangian transport by integrating the Eulerian mean flow and Stokes drift velocity. We also carry out new particle tracking velocimetry (PTV) experiments for wavepackets in a laboratory wave flume in the finite-depth regime, in which the effect of set-down on Lagrangian particle displacement becomes significant. We thus extend results for deep-water for which the set-down can be ignored, obtained by van den Bremer et al. [43], to finite depth for which it cannot. We measure the set-down, take into account the effect of error waves, extract the Lagrangian displacement and compare results with our theoretical predictions, finding good agreement.

The paper is laid out as follows. First, section II outlines second-order theory, leading to a solution for Eulerian return flow in arbitrary depth and displacements arising from Eulerian mean flow and Stokes drift. Section III describes the experimental method, and section IV presents a comparison of theoretically predicted and experimentally determined displacements. Finally, conclusions are drawn in section V.

\section{SECOND-ORDER THEORY}

\section{A. Governing equations}

We consider unidirectional waves propagating over a horizontal bed in the $(x, z)$-plane, with $z$ measured vertically upwards from the still water level, and the free surface located at $z=\eta$. For the irrotational flow of inviscid, incompressible fluid, the governing equation is Laplace,

$$
\nabla^{2} \phi=0 \quad \text { for } \quad-d \leq z \leq \eta
$$




\begin{tabular}{lccc}
\hline $\begin{array}{l}\text { Deep carrier wave and deep return } \\
\text { flow }\end{array}$ & $\gg 1$ & $\gg 1$ & $\begin{array}{c}\text { Dysthe (1979) } \\
\text { van den Bremer and Taylor (2016) } \\
\text { This paper }\end{array}$ \\
\hline $\begin{array}{l}\text { Deep carrier wave and shallow } \\
\text { return flow }\end{array}$ & $\gg 1$ & $\begin{array}{c}\text { Mei et al. (1989) } \\
\text { anguet-Higgins and Stewart (1962) } \\
\text { and Stewartson (1974) } \\
\text { Davey } \\
\text { Brinch-Nielsen and Jonnson (1986) } \\
\text { This paper }\end{array}$ \\
$\begin{array}{l}\text { Intermediate-depth carrier wave } \\
\text { and intermediate-depth return flow }\end{array}$ & $\mathcal{O}(1)$ & $\mathcal{O}(1)$ & This paper \\
\hline $\begin{array}{l}\text { Intermediate-depth carrier wave } \\
\text { and shallow return flow }\end{array}$ & $\mathcal{O} 1)$ & $\ll 1$ & $\begin{array}{c}\text { Longuet-Higgins and Stewart (1962) } \\
\text { Davey and Stewartson (1974) } \\
\text { Longuet-Higgins and Stewart (1962) } \\
\text { This paper }\end{array}$ \\
\hline
\end{tabular}

Table I. Classification of the literature in different regimes based on depth relative to the carrier wave length $\left(k_{0} d\right)$ and depth relative to the wavepacket length or the return flow scale $(d / \sigma)$.

where $\phi$ is the velocity potential and $d$ depth. Equation (1) is solved subject to the no-flow bottom boundary condition,

$$
\frac{\partial \phi}{\partial z}=0 \quad \text { at } \quad z=-d
$$

and the kinematic and dynamic linear free surface boundary conditions,

$$
w-\frac{\partial \eta}{\partial t}-u \frac{\partial \eta}{\partial x}=0 \quad \text { and } \quad g \eta+\frac{\partial \phi}{\partial t}+\frac{1}{2}(\nabla \phi)^{2}=0 \quad \text { at } \quad z=\eta,
$$

where $t$ is time, $g$ is gravity, and the velocity components are $u=\partial \phi / \partial x$ and $w=\partial \phi / \partial z$.

\section{B. Solutions using perturbation methods}

We begin by carrying out a Stokes expansion using the steepness $\alpha \equiv k_{0} a$, where $a$ is the maximum amplitude of the (linear) free surface $A_{0}$ and $k_{0}$ is the carrier wavenumber. Using a Taylor-series expansion of the free surface boundary conditions $(3 \mathrm{a}, \mathrm{b})$ about $z=0$, we obtain after some manipulation (see also $[45,46]$ ), utilizing the Laplace equation (1):

$$
\begin{gathered}
\left(\frac{1}{g} \frac{\partial^{2}}{\partial t^{2}}+\frac{\partial}{\partial z}\right) \phi^{(2)}=\overline{\frac{\partial}{\partial x}\left(\frac{\partial \phi^{(1)}}{\partial x} \eta^{(1)}\right)-\frac{1}{g} \frac{\partial}{\partial t}\left(\frac{1}{2}\left(\nabla \phi^{(1)}\right)^{2}+\frac{\partial^{2} \phi^{(1)}}{\partial z \partial t} \eta^{(1)}\right)}+\mathcal{O}\left(\alpha^{3}\right) \quad \text { at } \quad z=0, \\
\eta^{(2)}=\frac{-1}{g}\left(\frac{\partial \phi^{(2)}}{\partial t}+\overline{\frac{\partial^{2} \phi^{(1)}}{\partial z \partial t} \eta^{(1)}+\frac{1}{2}\left|\nabla \phi^{(1)}\right|^{2}}\right)+\mathcal{O}\left(\alpha^{3}\right) \quad \text { at } \quad z=0,
\end{gathered}
$$

where the superscripts denote the order in $\alpha$, and we only consider terms up to second order in $\alpha$.

Wavepackets can be created by modulating a single frequency carrier wave with angular frequency $\omega_{0}$ and wavenumber $k_{0}$. Assuming the length scale of the modulation $\sigma$ is much larger than the wavelength of the carrier wave, allows a multiple-scales expansion in the small parameter $\epsilon \equiv 1 /\left(k_{0} \sigma\right)$. The parameter $\epsilon$ is a measure of the bandwidth of the spectrum, and the leading-order terms we consider correspond to a narrow-banded spectrum centred around the carrier wave. Accordingly, we have the fast variables $x, z$ and $t$ and the slow variable $X=\epsilon\left(x-c_{g, 0} t\right)$, where $c_{g, 0} \equiv \partial \omega /\left.\partial k\right|_{k=k_{0}}=\omega_{0} / k_{0}\left(1 / 2+k_{0} d / \sinh \left(2 k_{0} d\right)\right)$ is the group velocity of the wavepacket. Our focus is on leadingorder terms; we thus ignore dispersion of the group and its associated higher-order scales. The fast variables allow change on the length scale of the carrier wave, whilst the slow variables represent changes in the modulation of the wavepacket. Derivatives are then expanded for fast and slow variables as

$$
\frac{\partial}{\partial x} \rightarrow \partial_{x}+\epsilon \partial_{X}
$$




\begin{tabular}{lcc} 
Linear field & $\mathcal{O}\left(\alpha \epsilon^{0}\right)$ & $\mathcal{O}\left(\alpha \epsilon^{1}\right)$ \\
\hline surface elevation $\eta^{(1)}$ & $A_{0}$ & $\imath \frac{k_{0} d}{k_{0} \tanh \left(k_{0} d\right)} \epsilon A_{0, X}$ \\
velocity potential $\phi^{(1)}$ & $-\imath \frac{c_{p, 0}}{k_{0}} A_{0} \hat{\eta}_{0}^{\prime}$ & $\frac{c_{p, 0}}{k^{2}} \epsilon A_{0, X}\left(\hat{\eta}_{1}^{\prime}+\left(2-c_{g, 0} / c_{p, 0}\right) \hat{\eta}_{0}^{\prime}\right)$ \\
horizontal velocity $u^{(1)}$ & $c_{p, 0} A_{0} \hat{\eta}_{0}^{\prime}$ & $\imath \frac{c_{p, 0}}{k_{0}} \epsilon A_{0, X}\left(\hat{\eta}_{1}^{\prime}+\left(1-c_{g, 0} / c_{p, 0}\right) \hat{\eta}_{0}^{\prime}\right)$ \\
vertical velocity $w^{(1)}$ & $-\imath \omega_{0} A_{0} \hat{\eta}_{0}$ & $c_{p, 0} \epsilon A_{0, X}\left(\hat{\eta}_{1}-c_{g, 0} / c_{p, 0} \hat{\eta}_{0}\right)$ \\
vertical displacement $\zeta^{(1)}$ & $A_{0} \hat{\eta}_{0}$ & $\imath \frac{1}{k_{0}} \epsilon A_{0, X} \hat{\eta}_{1}$ \\
horizontal displacement $\xi^{(1)}$ & $\imath \frac{1}{k_{0}} \hat{\eta}_{0}^{\prime}$ & $-\frac{1}{k_{0}^{2}} \epsilon A_{0, X}\left(\hat{\eta}_{1}^{\prime}+\hat{\eta}_{0}^{\prime}\right)$
\end{tabular}

Table II. Expressions for the different linear (in $\left.\alpha \equiv k_{0}\left|a_{0}\right|\right)$ fields at $\mathcal{O}\left(\alpha^{1} \epsilon^{0}\right)$ and $\mathcal{O}\left(\alpha^{1} \epsilon^{1}\right)$. The actual fields are the real parts of the tabulated expressions after multiplication by $\exp \left[\imath\left(k_{0} x-\omega_{0} t\right)\right]$.

From here on, $\partial / \partial x$ will be used to denote combined slow and fast derivatives, whereas $\partial_{x}$ and $\partial_{X}$ denote fast and slow derivatives, respectively. The overlines in (4-5) correspond to averaging over the fast scales, and we ignore any superharmonic contributions to the second-order terms, as they do not contribute to the mean flow. We will now consider the first two orders in $\alpha$ in turn.

\section{First-order in steepness $\mathcal{O}(\alpha)$}

Similar to [21, 22], we write the $\mathcal{O}(\alpha)$ solutions as summations over different orders in $\epsilon$ (see also [47]),

$$
\begin{aligned}
& \zeta^{(1)}=\left\{A_{0}(X) \hat{\zeta}_{0}(z)+\epsilon A_{1}(X) \hat{\zeta}_{1}(z)\right\} e^{\imath\left(k_{0} x-\omega_{0} t\right)}+\mathcal{O}\left(\alpha \epsilon^{2}\right), \\
& \phi^{(1)}=\left\{B_{0}(X) \hat{\phi}_{0}(z)+\epsilon B_{1}(X) \hat{\phi}_{1}(z)\right\} e^{\imath\left(k_{0} x-\omega_{0} t\right)}+\mathcal{O}\left(\alpha \epsilon^{2}\right),
\end{aligned}
$$

where the subscripts denote the order in $\epsilon$ and we only consider the first two orders. The variable $\zeta^{(1)}$ denotes the linear vertical displacement field, which corresponds to the linear free surface elevation $\eta^{(1)}$ evaluated at $z=0$, namely $\eta^{(1)}=\zeta^{(1)}(z=0)$, and can be found from $\partial \zeta^{(1)} / \partial t=w^{(1)}$ (cf. (3a)). The vertical structure functions $\hat{\zeta}_{0}(z), \hat{\zeta}_{1}(z)$, $\hat{\phi}_{0}(z)$ and $\hat{\phi}_{1}(z)$ have to be chosen so that the Laplace equation (1) and the bottom boundary condition (2) are satisfied. We find that

$$
\hat{\zeta}_{0}(z)=\frac{\sinh \left(k_{0}(d+z)\right)}{\sinh \left(k_{0} d\right)}, \quad \hat{\zeta}_{1}(z)=a_{0} \sinh \left(k_{0}(d+z)\right)-\frac{k(d+z) \cosh \left(k_{0}(d+z)\right)}{\sinh \left(k_{0} d\right)},
$$

and

$$
\hat{\phi}_{0}(z)=\frac{\cosh \left(k_{0}(d+z)\right)}{\sinh \left(k_{0} d\right)}, \quad \hat{\phi}_{1}(z)=a_{0} \cosh \left(k_{0}(d+z)\right)-\frac{k_{0}(d+z) \sinh \left(k_{0}(d+z)\right)}{\sinh \left(k_{0} d\right)}+\left(1-c_{g, 0} / c_{p, 0}\right) \frac{\cosh \left(k_{0}(d+z)\right)}{\sinh \left(k_{0} d\right)}
$$

where $a_{0}$ can be freely chosen (cf. homogeneous solution) and we set $a_{0}=0$. Table II reports all the $\mathcal{O}(\alpha)$ polarization relationships expressed in terms of the amplitude envelope of the free surface at $\mathcal{O}\left(\alpha \epsilon^{0}\right)$.

From the linear solution, we can directly evaluate the Stokes drift, which is generally defined by:

$$
\mathbf{u}_{\mathrm{S}} \equiv \overline{\frac{\partial \mathbf{u}^{(1)}}{\partial x} \xi^{(1)}+\frac{\partial \mathbf{u}^{(1)}}{\partial z} \zeta^{(1)}}
$$

where $\xi^{(1)}$ and $\zeta^{(1)}$ are the linear horizontal and vertical displacements (cf. $\left.\partial \xi^{(1)} / \partial t=u^{(1)}, \partial \zeta^{(1)} / \partial t=w^{(1)}\right)$. Using the linear polarization relationships from table II, we can find explicit expressions for the horizontal and vertical Stokes drift velocities:

$$
u_{\mathrm{S}}=\frac{\omega_{0} k_{0}}{2} \frac{\cosh \left(2 k_{0}(d+z)\right)}{\sinh ^{2}\left(k_{0} d\right)}\left|A_{0}\right|^{2} \quad \text { and } \quad w_{\mathrm{S}}=-\frac{\omega_{0}}{4}\left(1+\frac{c_{g, 0}}{c_{p, 0}}\right) \frac{\sinh \left(2 k_{0}(z+d)\right)}{\sinh ^{2}\left(k_{0} d\right)} \epsilon \partial_{X}\left|A_{0}\right|^{2}
$$




\section{Second-order in steepness $\mathcal{O}\left(\alpha^{2}\right)$}

Seeking a solution for the Eulerian mean-flow potential $\phi^{(2)}$ that varies on the slow scale $X$ and using the linear polarization relationships in table II, equation (4) becomes,

$$
\begin{aligned}
\left(\frac{\epsilon^{2} c_{g, 0}^{2}}{g} \partial_{X X}+\partial_{z}\right) \phi^{(2)}(X, z) & =\epsilon \frac{\omega_{0} \partial_{X}\left|A_{0}\right|^{2}}{2 \tanh \left(k_{0} d\right)}+\epsilon c_{g, 0} \frac{\omega_{0}^{2} \partial_{X}\left|A_{0}\right|^{2}}{4 g \sinh ^{2}\left(k_{0} d\right)} \\
& =\quad \epsilon\left(1+C_{\mathrm{FD}}\left(k_{0} d\right)\right) \frac{\omega_{0} \partial_{X}\left|A_{0}\right|^{2}}{2 \tanh \left(k_{0} d\right)}
\end{aligned}
$$

where the coefficient $C_{\mathrm{FD}}\left(k_{0} d\right)$ represents terms that only contribute in finite depth:

$$
C_{\mathrm{FD}}\left(k_{0} d\right)=\frac{c_{g, 0} \omega_{0}}{g \sinh \left(2 k_{0} d\right)}=\frac{k_{0} d \operatorname{sech}^{2}\left(k_{0} d\right)+\tanh \left(k_{0} d\right)}{2 \sinh \left(2 k_{0} d\right)},
$$

and in deep-water $\left(k_{0} d \gg 1\right)$, we have $C_{\mathrm{FD}}\left(k_{0} d\right) \rightarrow 0$.

The forcing equation (13) can be solved using a Fourier transform to give:

$$
\phi^{(2)}=-\frac{\omega_{0}\left(1+C_{\mathrm{FD}}\left(k_{0} d\right)\right)}{4 \pi \tanh \left(k_{0} d\right)} \int_{-\infty}^{\infty} \widehat{\left|A_{0}\right|^{2}} \frac{\cosh (\kappa(z+d))}{\sinh (\kappa d)} \frac{\imath \exp (\imath \kappa \tilde{x})}{1-\frac{c_{g, 0}^{2} \kappa}{g \tanh (\kappa d)}} \mathrm{d} \kappa
$$

where $\widehat{\left|A_{0}\right|^{2}}$ is the Fourier transform of $\left|A_{0}\right|^{2}$ in $\tilde{x}$ and $\tilde{x} \equiv X / \epsilon=x-c_{g, 0} t$. The poles of this integral do not make a contribution and are discussed in appendix A. Using (15), the set-down can then be calculated from (5) to be:

$$
\eta^{(2)}=-\frac{\omega_{0}^{2}\left|A_{0}\right|^{2}}{4 g \sinh ^{2}\left(k_{0} d\right)}-\frac{\left.c_{g, 0} \omega_{0}\left(1+C_{\mathrm{FD}}\left(k_{0} d\right)\right)\right)}{2 g \tanh \left(k_{0} d\right)} \int_{-\infty}^{\infty} \kappa \widehat{\left|A_{0}\right|^{2}} \frac{\cosh (\kappa(z+d))}{\sinh (\kappa d)} \frac{\exp (\imath \kappa \tilde{x})}{1-\frac{c_{g, 0}^{2} \kappa}{g \tanh (\kappa d)}} \mathrm{d} \kappa
$$

When examining the left-hand side of the forcing equation (13), care must be taken with regard to the order of $\phi^{(2)}$ (see also the discussion in [48]). Here, $\kappa$ is the wavenumber associated with the wavepacket and scales with $\epsilon$. The order of $\phi^{(2)}$ then depends on the order of $k_{0} d$. If $k_{0} d$ is large (deep-water) and $\epsilon$ is small, in such a manner that $d / \sigma=\epsilon k_{0} d \gg 1$, it is justified to make the 'rigid-lid' approximation by neglecting the set-down. In intermediate depth and with a sufficiently narrow-banded wavepacket, the return flow must be shallow $d / \sigma=\mathcal{O}(\epsilon) \ll 1($ i.e. $\epsilon=1 /\left(k_{0} \sigma\right)$ implies $d / \sigma=k_{0} d \epsilon=\mathcal{O}(\epsilon)$ when $\left.k_{0} d=\mathcal{O}(1)\right)$. Thus, the small-argument approximations of hyperbolic functions can be used (i.e. $\cosh (\kappa d) / \sinh (\kappa d) \rightarrow 1 /(\kappa d)$ ). As a result, $\phi^{(2)}$ becomes one order more significant in $\epsilon$ (i.e. $\left.\left.\left.\phi^{(2)}\right|_{k_{0} d=\mathcal{O}(1)} \propto \epsilon^{-1} \phi^{(2)}\right|_{k_{0} d \gg 1}\right)$. If $k_{0} d$ is $\mathcal{O}(1)$, the inclusion of $\partial_{X X} \phi^{(2)}$ in the left-hand side of the forcing equation is therefore required (this term was incorrectly ignored in [44]).

The relative depth of the return flow $(d / \sigma)$ can be assumed to be shallow or deep, allowing the integrals in (16) and (15) to be calculated analytically. Our solution for general $d / \sigma$ can then be manipulated to recover the results of Longuet-Higgins and Stewart [3] and Mei et al. [23] for $d / \sigma \ll 1$ (shallow return flow), and Dysthe [24] and van den Bremer and Taylor [25] for $d / \sigma \gg 1$ (deep return flow) (see appendix B).

\section{Lagrangian displacements}

\section{Arbitrary water depth: $k_{0} d=\mathcal{O}(1)$ and $d / \sigma=\mathcal{O}(1)$}

The Lagrangian velocity under a wavepacket comprises the Stokes drift and the Eulerian return flow, so that $\Delta x_{\mathrm{L}}=\Delta x_{\mathrm{S}}+\Delta x_{\mathrm{E}}$. To allow for comparison to experiments in which displacements have to be calculated over finite times to avoid the effect of error waves and their reflections, we calculate finite-time Lagrangian displacements. The Stokes drift (12) can be readily integrated between $t_{1}$ and $t_{2}$ to give:

$$
\Delta x_{\mathrm{S}}=\frac{\omega_{0} k_{0}}{2} \frac{\cosh \left(2 k_{0}(d+z)\right)}{\sinh ^{2}\left(k_{0} d\right)} \int_{t_{1}}^{t_{2}}\left|A_{0}\right|^{2} \mathrm{~d} t
$$




$$
\Delta z_{\mathrm{S}}=-\frac{1}{4}\left(1+\frac{c_{p, 0}}{c_{g, 0}}\right) \frac{k_{0} \sinh \left(2 k_{0}(z+d)\right)}{\sinh ^{2}\left(k_{0} d\right)}\left(\left|A_{0}\right|_{t=t_{2}}^{2}-\left|A_{0}\right|_{t=t_{1}}^{2}\right)
$$

In arbitrary water depth, making no assumptions about the scale of the group relative to the depth $d / \sigma$ and thus about the depth of the return flow, the horizontal and vertical components of the Eulerian return flow are obtained by differentiating (15); the displacements are then be obtained by integrating over time:

$$
\begin{aligned}
& \Delta x_{\mathrm{E}}=-\frac{\omega_{0}\left(1+C_{\mathrm{FD}}\left(k_{0} d\right)\right)}{4 \pi \tanh \left(k_{0} d\right)} \int_{t_{1}}^{t_{2}} \int_{-\infty}^{\infty} \kappa \widehat{\left|A_{0}\right|^{2}} \frac{\cosh (\kappa(z+d))}{\sinh (\kappa d)} \frac{\exp (\imath \kappa \tilde{x})}{1-\frac{c_{g, 0}^{2} \kappa}{g \tanh (\kappa d)}} \mathrm{d} \kappa \mathrm{d} t, \\
& \Delta z_{\mathrm{E}}=-\frac{\omega_{0}\left(1+C_{\mathrm{FD}}\left(k_{0} d\right)\right)}{4 \pi \tanh \left(k_{0} d\right)} \int_{t_{1}}^{t_{2}} \int_{-\infty}^{\infty} \kappa \widehat{\left|A_{0}\right|^{2}} \frac{\sinh (\kappa(z+d))}{\sinh (\kappa d)} \frac{\imath \exp (\imath \kappa \tilde{x})}{1-\frac{c_{g, 0}^{2} \kappa}{g \tanh (\kappa d)}} \mathrm{d} \kappa \mathrm{d} t .
\end{aligned}
$$

\section{Shallow return flow: $k_{0} d=\mathcal{O}(1)$ and $d / \sigma \ll 1$}

Assuming a shallow return flow $(d / \sigma \ll 1)$ enables an explicit solution of the integral over wavenumber in $(15)$ to be obtained (using the small-argument approximation of the hyperbolic tangent). In that case, the Eulerian flow velocity components become:

$$
u_{\mathrm{E}}=-\frac{\omega_{0}\left(1+C_{\mathrm{FD}}\left(k_{0} d\right)\right)}{2 \tanh \left(k_{0} d\right) d\left(1-\frac{c_{g, 0}^{2}}{g d}\right)}\left|A_{0}\right|^{2} \quad \text { and } \quad w_{\mathrm{E}}=-\frac{\omega_{0}\left(1+C_{\mathrm{FD}}\left(k_{0} d\right)\right)}{2 \tanh \left(k_{0} d\right)\left(1-\frac{c_{g, 0}^{2}}{g d}\right)} \frac{(z+d)}{d} \partial_{X}\left|A_{0}\right|^{2} .
$$

Integrating the horizontal Eulerian velocity when the return flow is shallow (21a) and combining with the previously derived horizontal displacement by the Stokes drift (17), which is unchanged, we obtain the horizontal Lagrangian displacement over the time interval $t_{1}$ to $t_{2}$,

$$
\Delta x_{\mathrm{L}}=\frac{\omega_{0} k_{0}}{2}\left[\frac{\cosh \left(2 k_{0}(z+d)\right)}{\sinh ^{2}\left(k_{0} d\right)}-\frac{\left(1+C_{\mathrm{FD}}\left(k_{0} d\right)\right)}{k_{0} \tanh \left(k_{0} d\right) d\left(1-\frac{c_{g, 0}^{2}}{g d}\right)}\right] \int_{t_{2}}^{t_{1}}\left|A_{0}\right|^{2} \mathrm{~d} t
$$

where $t_{1}$ and $t_{2}$ are arbitrary time values. If the group is Gaussian and of the form $A_{0}=a_{0} \exp \left(-\left(x-c_{g, 0} t\right)^{2} / 2 \sigma^{2}\right)$ and the time integral is taken over the entire group, such that $t_{1} \rightarrow-\infty$ is before the arrival of the group and $t_{2} \rightarrow \infty$ after the group has passed, then we obtain:

$$
\Delta x_{\mathrm{L}}=\frac{\sqrt{\pi} \omega_{0} \alpha^{2} \sigma}{2 k_{0} c_{g, 0}}\left[\frac{\cosh \left(2 k_{0}(z+d)\right)}{\sinh ^{2}\left(k_{0} d\right)}-\frac{\left(1+C_{\mathrm{FD}}\left(k_{0} d\right)\right)}{k_{0} \tanh \left(k_{0} d\right) d\left(\left(1-\frac{c_{g, 0}^{2}}{g d}\right)\right.}\right] .
$$

The vertical Lagrangian displacement component between times $t_{1}$ and $t_{2}$ is

$$
\Delta z_{\mathrm{L}}=\left[\frac{1}{4}\left(1+\frac{c_{p, 0}}{c_{g, 0}}\right) \frac{k_{0} \sinh \left(2 k_{0}(z+d)\right)}{\sinh ^{2}\left(k_{0} d\right)}-\frac{\omega_{0}\left(1+C_{\mathrm{FD}}\left(k_{0} d\right)\right)}{2 c_{g, 0} \tanh \left(k_{0} d\right)\left(1-\frac{c_{g, 0}^{2}}{g d}\right)} \frac{z+d}{d}\right]\left(\left|A_{0}\right|_{t=t_{2}}^{2}-\left|A_{0}\right|_{t=t_{1}}^{2}\right),
$$

from which we can obtain for the maximum or minimum displacement underneath the centre of the group,

$$
\Delta z_{\mathrm{L}}=\left[\frac{1}{4}\left(1+\frac{c_{p, 0}}{c_{g, 0}}\right) \frac{\sinh \left(2 k_{0}(z+d)\right)}{\sinh ^{2}\left(k_{0} d\right)}-\frac{\omega_{0}\left(1+C_{\mathrm{FD}}\left(k_{0} d\right)\right)}{2 k_{0} c_{g, 0} \tanh \left(k_{0} d\right)\left(1-\frac{c_{g, 0}^{2}}{g d}\right)} \frac{z+d}{d}\right] \frac{\alpha^{2}}{k_{0}} .
$$

Figure 1 now examines the effect of ignoring the set-down when computing displacements. As the relative depth $k_{0} d$ becomes shallower, the role of the set-down increases, and the continuous lines, which include the effect of the setdown, diverge from the dashed lines, which ignore this effect. This paper sets out to observe this effect experimentally. 
(a)

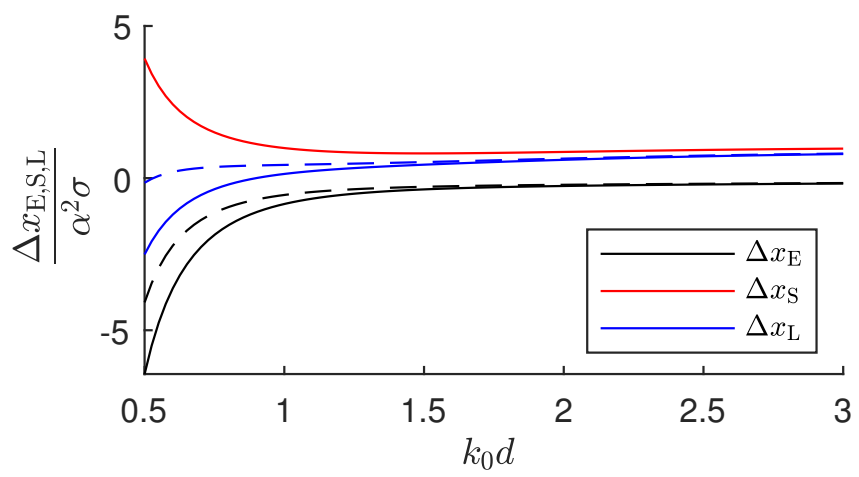

(b)

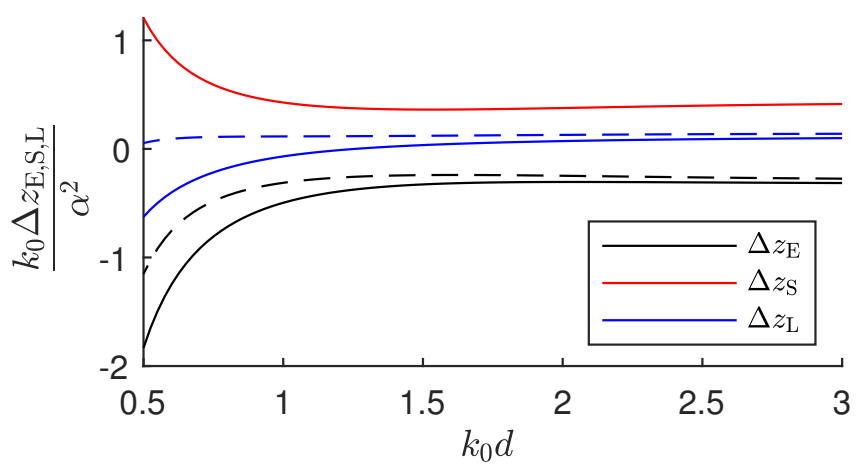

Figure 1. Theoretical normalized horizontal (left panel) and vertical (right panel) displacements as a function of the relative depth $k_{0} d$ at $z=0$ (from (23) and (25)). The displacements by the Eulerian mean flow displacement are shown as black lines, the displacements by the Stokes drift by red lines and the Lagrangian displacements by blue lines. The solid lines show the displacements when the set-down is fully accounted for, whereas the dashed lines ignore the set-down.

\section{EXPERIMENTAL METHODOLOGY}

\section{A. Set-up and data acquisition}

To validate the predicted particle trajectories under a wavepacket, a series of particle tracking velocimetry (PTV) experiments were undertaken in the Sediment Wave Flume in the Coastal, Ocean and Sediment Transport (COAST) Laboratory, University of Plymouth, UK. The flume has a length of $35 \mathrm{~m}$, a width of $0.60 \mathrm{~m}$, and was filled with water to a depth of $0.50 \mathrm{~m}$, as shown in fig. 2. We used a double-element piston-type wavemaker to generate a focused wavepacket with a spectral shape that linearly focuses to a Gaussian packet $A_{0}=a_{0} \exp \left(-\left(x_{f}-c_{g, 0} t\right)^{2} / 2 \sigma^{2}\right)$ at a measurement zone located $x_{f}=19.75 \mathrm{~m}$ from the resting position of the wavemaker. Seven resistance-type wave gauges provided $128 \mathrm{~Hz}$ free surface elevation measurements. A light-emitting diode (LED) light box illuminated the motions of small near-neutrally buoyant particles in a plane, which were captured by a camera positioned outside the flume. After propagating through the measurement zone, the dispersed wavepackets were absorbed by meshfilled wedges within an 'absorption zone' located at the downstream end of the wave flume. The distance to the measurement zone was prescribed to allow as much separation as possible of the sub-harmonic error wave from the group, whilst also avoiding reflections. To ensure near-quiescent initial conditions for each experiment, the water surface was allowed to settle for 10 minutes between experiments. The settling time of 10 minutes was short enough that the slightly positively-buoyant particles remained evenly distributed throughout the water column at the start of each experiment.

Fluid trajectories were measured by tracking a large number of illuminated small-diameter seeding particles within the flow. These 'Plascoat' particles were approximately $150 \mu \mathrm{m}$ in diameter, small enough to be considered dynamically unimportant. Although the particles were slightly positively buoyant, their upward settling velocities were negligible compared to their motion beneath the free surface waves. The particles were effectively uniformly mixed throughout the measurement zone, which was achieved by adding new particles between experiments when necessary, mixing up the water column and waiting for the flume to become quiescent before starting experiments. Due to the light sheet, only particles in a thin slice in the $(x-z)$-plane were tracked. The camera field of view was not able to capture the full depth of the flume. Thus, to obtain a complete coverage of water column, the same experiment was repeated three times. Ambient lighting was removed whilst a Photron SA4 high-speed camera captured the particle motions at $125 \mathrm{frames} / \mathrm{s}$, at a resolution of 1024 by 1024 pixels and a shutter speed of $1 / 125 \mathrm{~s}$. This shutter speed achieved an acceptable compromise between the requirements of obtaining very sharp images and allowing sufficient light to enter the camera during each image exposure. An image of a ruler was used to quantify the pixel scale, and distortion was found to be negligible for these tests. 


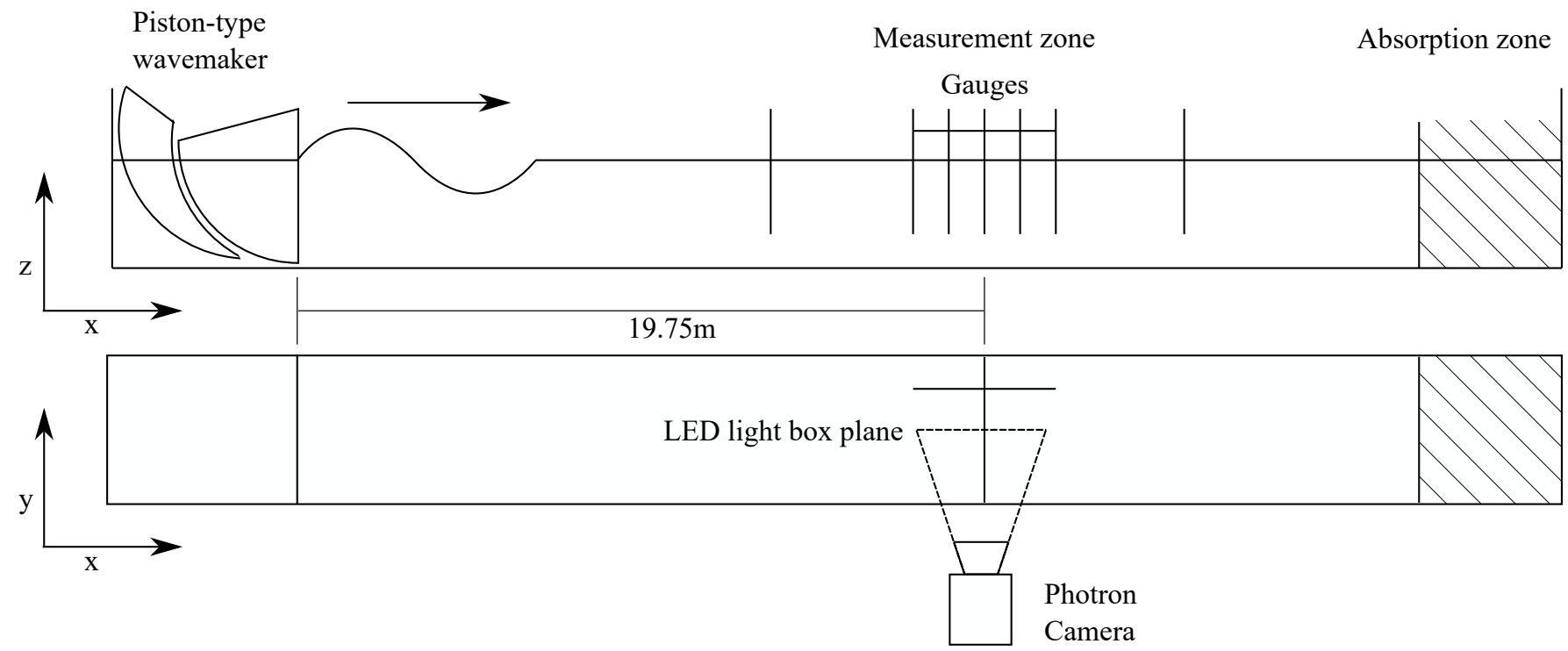

Figure 2. Experimental setup used to obtain PTV measurements of fluid trajectories beneath focused wavepackets generated by a double-element piston-type wave maker at the COAST laboratory, University of Plymouth, UK.

\section{B. Wave generation}

If operated using a first-order signal, a wavemaker will generate spurious error waves (see e.g. [16, 18] for a discussion on the effects of these error waves). To partially eliminate the generation of these error waves, the secondorder corrections of Schäffer [19] were applied to the wavemaker signal. The Edinburgh Designs Ltd (EDL) wavemaker differs from an idealised piston-type wave maker in two ways. First, the EDL wavemaker is a double-element paddle, which does not extend to the base of the flume. Second, the paddle does not have the range of movement required to eliminate sub-harmonic error waves in finite water depth. As a result, error waves were not completely removed by the correction method (see also [49]). Whittaker et al. [17] concluded that the sub-harmonic error wave amplitude was reduced by approximately $60 \%$ in similar conditions. In our case, sub-harmonic error waves had relatively large amplitude due to the finite-depth nature of experiments (finite $k_{0} d$ ), and caused significant difficulty when calculating the displacements discussed in section IV A.

\section{Matrix of experiments}

Table III lists the parameters for the 9 experiments we conducted, varying the relative water depth $k_{0} d$ and the bandwidth $\epsilon$. Frequencies were chosen such that the water depth is 'intermediate' $\left(k_{0} d \approx 1-3\right)$; amplitudes such that second-order motions are large enough to measure, but not so large so that effects beyond second-order play a role; and the bandwidth parameter $\epsilon=1 /\left(k_{0} \sigma\right)$ such that the wavepacket is still quasi-monochromatic, but not so long that reflections play a role.

\section{Data processing and removal of background motion}

The free surface was measured using seven resistance wave gauges, five spread around the focus location and two significant distances before and after the focus location. The wavepackets were deliberately chosen to have a narrowbanded spectrum to allow frequency filtering to separate the linear and second-order sub-harmonic parts from the wave gauge signal. This was achieved using a low-pass filter of $0.5 f_{0}$ to separate the sub-harmonic set-down and a band-pass filter between $0.7 f_{0}$ and $1.3 f_{0}$ to extract the linear free surface elevation. The linear free surface elevation is shown in fig. 3, along with the measured envelope $\left|A_{0}\right|$. The measured envelope is calculated using the Hilbert transform of the linear free surface elevation, which in turn is used to predict the mean flow using (22). The use of the measured envelope at the location where the trajectories are measured accounts for any dissipation or non-linear 
(a)

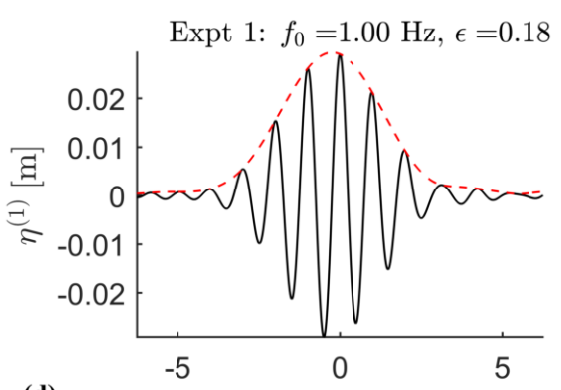

(d)

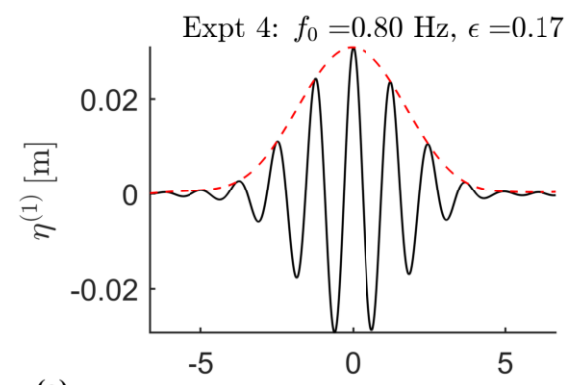

(g)

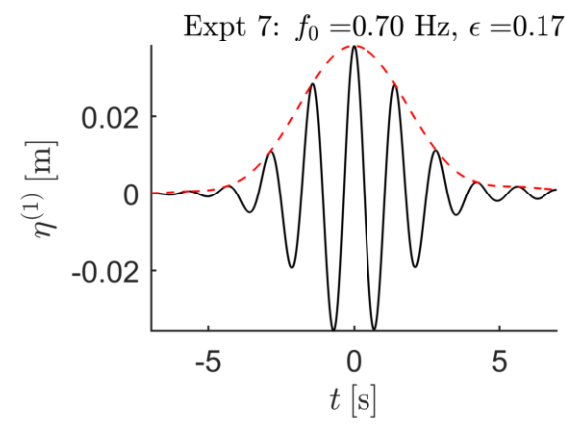

(b)

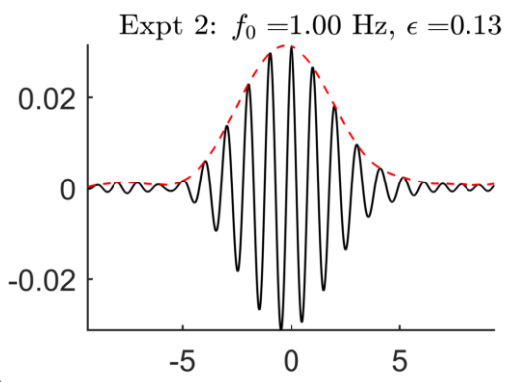

(e)

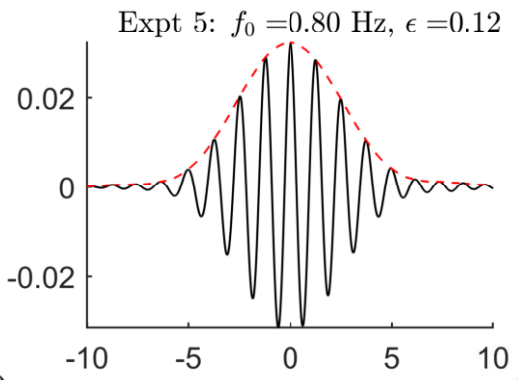

(h)

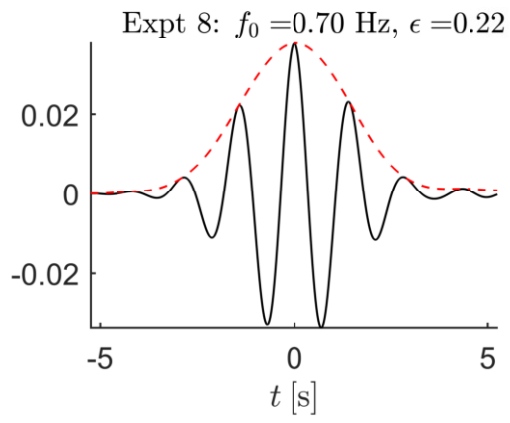

(c)

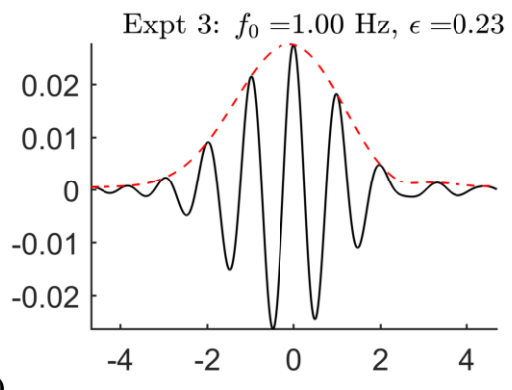

(f)

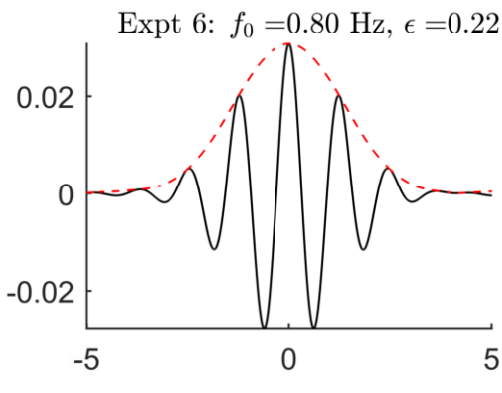

(i)

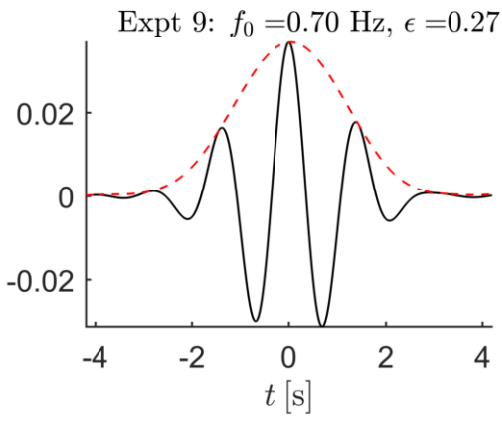

Figure 3. The experimental linear free surface elevation measured at the focus location (black lines). The linear part has been extracted with a band-pass filter between $0.7 f_{0}$ and $1.3 f_{0}$. The envelope (red dashes lines) has been calculated using a Hilbert transform of the linear free surface.

dispersion, which may occur between the wavemaker and the measurement zone.

The recorded images were processed using Streams software, a specialist package for flow visualisation applications [50]. Particles within each image were identified using a dual threshold algorithm, which first searched for pixels of intensity greater than a threshold value, identified the maximum pixel intensity within the group of pixels, and finally defined particle boundaries based on a fraction of this maximum intensity. The identified particles were subsequently matched between frames using an 'auction' optimisation algorithm, which minimised the total cost of all potential matches for each successive pair of frames, where the cost was first assigned based on particle distance, then based on matched particle velocities in a small region. Recent velocities of matched particles were finally used to generate additional matches where appropriate. Lagrangian paths were then calculated and stored for post-processing.

Background motion and the motion due to the sub-harmonic error wave had to be eliminated in order to calculate the displacement due to Stokes drift and Eulerian return flow. The background motion is much slower than the desired measured Lagrangian motion, and consists of a combination of a rise velocity of the particles, long waves that decay slowly in the flume, circulation induced by small air flows over the water surface, which cannot be eliminated 


\begin{tabular}{ccccc} 
Experiment Peak frequency Steepness & $\begin{array}{c}\text { Bandwidth } \\
f_{0}[\mathrm{~Hz}]\end{array}$ & $\begin{array}{c}\text { Relative depth } \\
\alpha=k_{0} a_{0}\end{array} \quad \begin{array}{c}\epsilon=\left(k_{0} \sigma\right)^{-1} \\
k_{0} d\end{array}$ \\
\hline 1 & 1.00 & 0.12 & 0.18 & 2.08 \\
2 & 1.00 & 0.13 & 0.13 & 2.08 \\
3 & 1.00 & 0.12 & 0.23 & 2.08 \\
4 & 0.80 & 0.09 & 0.17 & 1.44 \\
5 & 0.80 & 0.09 & 0.12 & 1.44 \\
6 & 0.80 & 0.09 & 0.22 & 1.44 \\
7 & 0.70 & 0.09 & 0.17 & 1.18 \\
8 & 0.70 & 0.09 & 0.22 & 1.18 \\
9 & 0.70 & 0.09 & 0.27 & 1.18
\end{tabular}

Table III. Matrix of experiments.

in the laboratory and mixing induced motion. The Stokes rise velocity of the spherical particles can be estimated as $w=(2 / 9)\left(\left(\rho_{w}-\rho_{p}\right) / \rho_{w}\right)\left(g R^{2} / \nu\right)=0.49 \mathrm{~mm} / \mathrm{s}$, where we have used $\rho_{p}=0.96 \mathrm{~g} / \mathrm{cm}^{3}$ (density of Plascoat particle), $\rho_{w}=1.00 \mathrm{~g} / \mathrm{cm}^{3}$ (density of water), $\nu=1.0 \times 10^{-6} \mathrm{~m}^{2} / \mathrm{s}$ (viscosity of water) and $R=75 \times 10^{-6} \mathrm{~m}$ (radius of Plascoat particle). This is much slower than the motion induced by the waves. The smallest amplitude of wave induced motion at focus are $u_{S}=17.7 \mathrm{~mm} / \mathrm{s}$ and $U_{E}=6.7 \mathrm{~mm} / \mathrm{s}$, which are significantly larger than the rise velocity of $0.49 \mathrm{~mm} / \mathrm{s}$. This permitted a suitable curve fit to the trajectories before and/or after the focused wavepacket to be subtracted from the original $x(\mathrm{t})$ and $z(\mathrm{t})$ trajectories. The most successful fitting method was then selected by studying the frequency spectrum of the trajectories after the curve fit had been subtracted. The exact procedure is described in appendix C.

\section{RESULTS}

\section{A. The sub-harmonic free surface elevation: set-down and error waves}

Figure 4 shows the experimental second-order sub-harmonic free surface elevation time series, calculated by filtering the free surface elevation at $0.5 f_{0}$. Each experiment clearly exhibits a set-down at the centre of the group $(t=0)$. Close to focus, the set-down matches the theoretical curve well, the maximum of the experimental setdown is very close to the theoretical. However, 'shoulders' can be seen either side of the experimental set-down. These shoulders are not to be confused with the predicted set-down shape in non-shallow return flow. The left shoulder corresponds to the sub-harmonic error wave propagating in the positive $x$-direction ahead of the group (and its bound set-down), and the right shoulder to its reflection travelling in the opposite direction. Because of its length relative to the water depth, the sub-harmonic error wave travels at the shallow water speed $(\sqrt{g d})$.

Figure 5 shows the time series of the sub-harmonic wave components at seven wave gauges plotted with an offset proportional to their respective distance from the focus location. Straight lines denote the propagation speeds of the different features: the positive group speed $c_{g, 0}$ for the set-down, the positive shallow-water speed $\sqrt{g d}$ for the sub-harmonic error wave (left shoulder) and the negative shallow-water speed $-\sqrt{g d}$ for its reflection (right shoulder). The error wave is larger and less separated in the shallower experiments (experiment 4-9 in fig. 4 and fig. 5), which is as expected because the second-order signal given to the paddle is produced less well and the error wave is consequently larger, and the difference between the group and the shallow-water speeds is smaller in these cases.

Based on the three repeats we carried out for each experiment, we can quantify the repeatability and quantify the measurement error associated with the (small) set-down. The left panel of fig. 6 shows an example of this for experiment 5 (where $f_{0}=0.80 \mathrm{~Hz}$ and $\epsilon=0.12$ ), with the three repeats plotted as a function of time along with the mean and confidence region of \pm 2 standard deviations. The set-down of the three experiments show remarkable repeatability, even though the maximum value of the set-down is only $1.2 \mathrm{~mm}$. The grey confidence band of \pm 2 standard deviations captures the theoretically predicted magnitude of the set-down (dashed red line), though there is an error in the time structure and alignment. The right panel of fig. 6 shows the measured magnitude of the set-down as a function of the theoretically predicted value for all experiments, with the $45^{\circ}$-line corresponding to perfect agreement. The error bars in this panel are not estimated from repeats but from the (absolute) calibration error of the gauges, giving generally larger confidence bands than the error estimated from repeats. A good agreement between theory and experiments is generally evident for the magnitude of the set-down underneath the centre of the group, where the error waves have not reached for all experiments. 


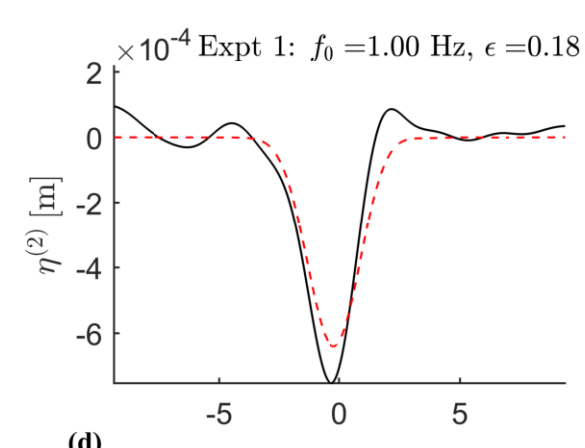

(d)

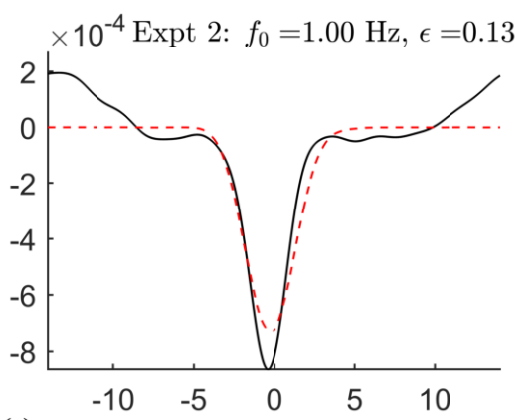

(e)
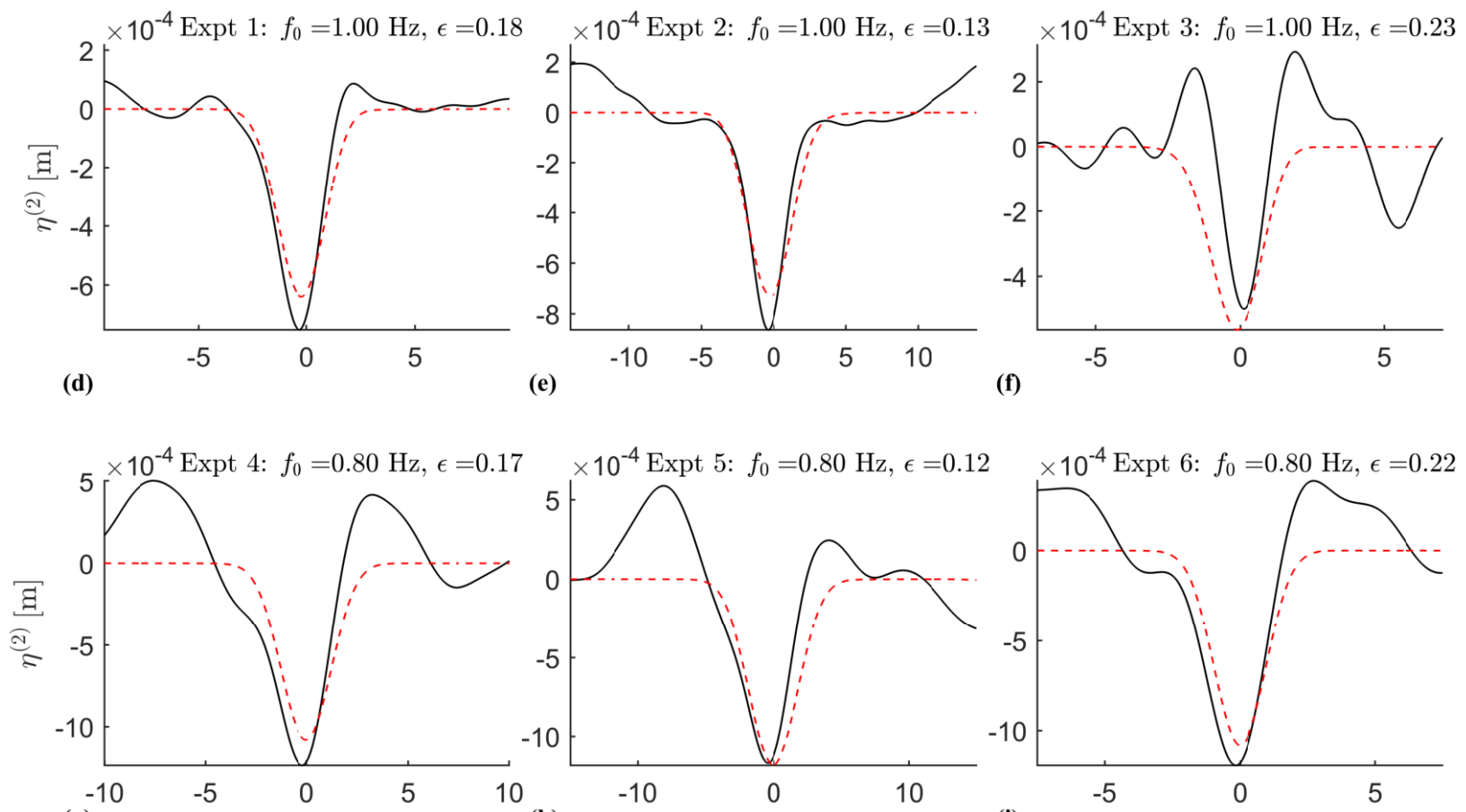

(g)

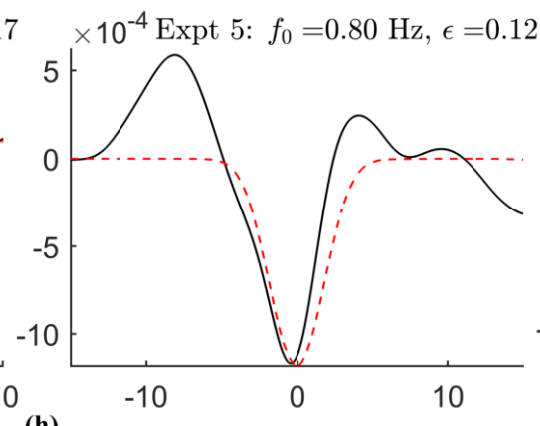

(h)
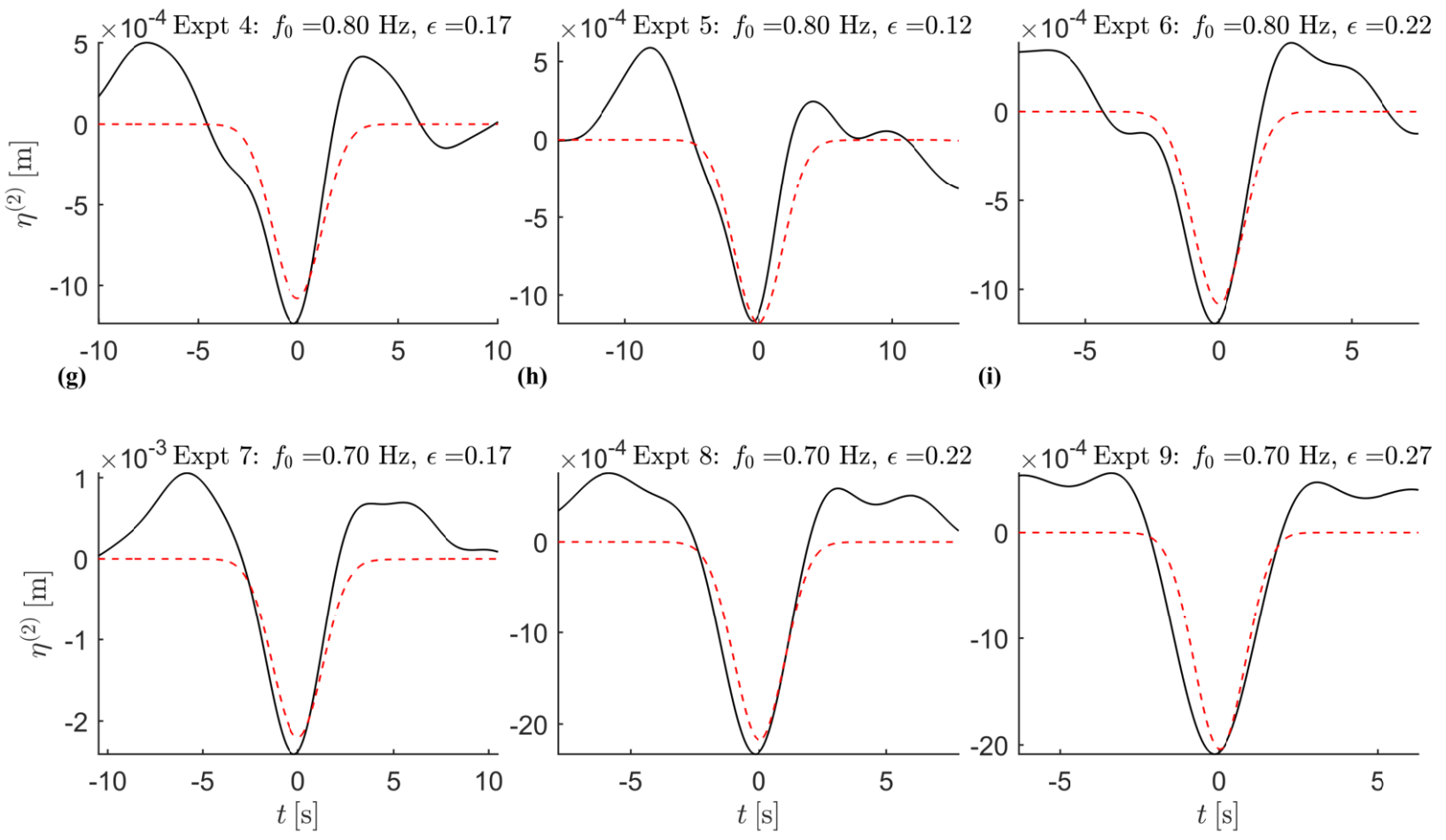

Figure 4. Time series of the sub-harmonic components of the free surface elevation representing a 'set-down', from experiments (solid black lines), extracted from the free surface elevation by a low-pass filter at $0.5 f_{0}$, and theory (dashed red lines) for the different experiments.

In the same way that the return flow underneath the set-down leads to a negative displacement of particles, the subharmonic error wave (a set-up travelling to the right) gives rise to positive displacement of particles, and its reflection (a set-up travelling to the left) to negative displacement. In experiments 4-9, the error wave did not separate out from the group before the group arrived at the focus location. Instead of calculating the true net displacement, in all experiments a small window $\left(t_{1}=-0.1 \sigma / c_{g, 0}, t_{2}=0.1 \sigma / c_{g, 0}\right)$ has been used to calculate the horizontal displacement to avoid including any error wave in the displacement. See appendix $\mathrm{C}$ for more detail. 


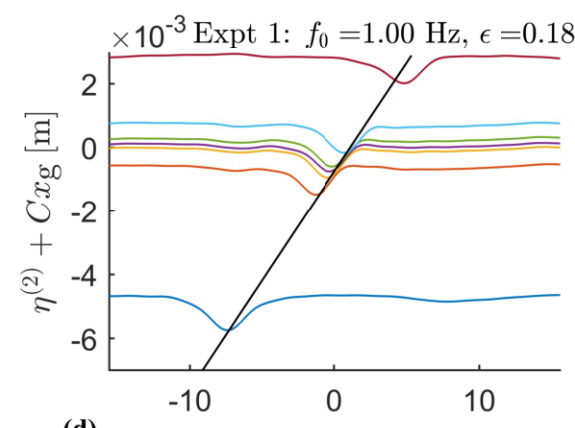

(d)

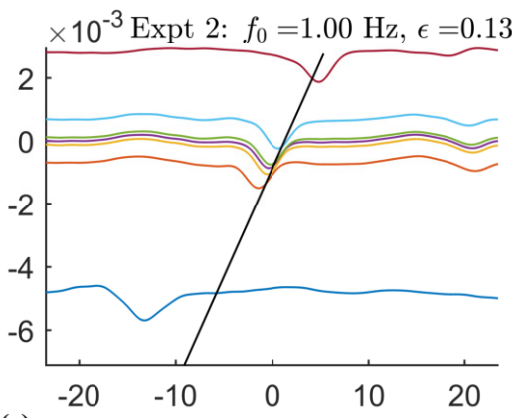

(e)
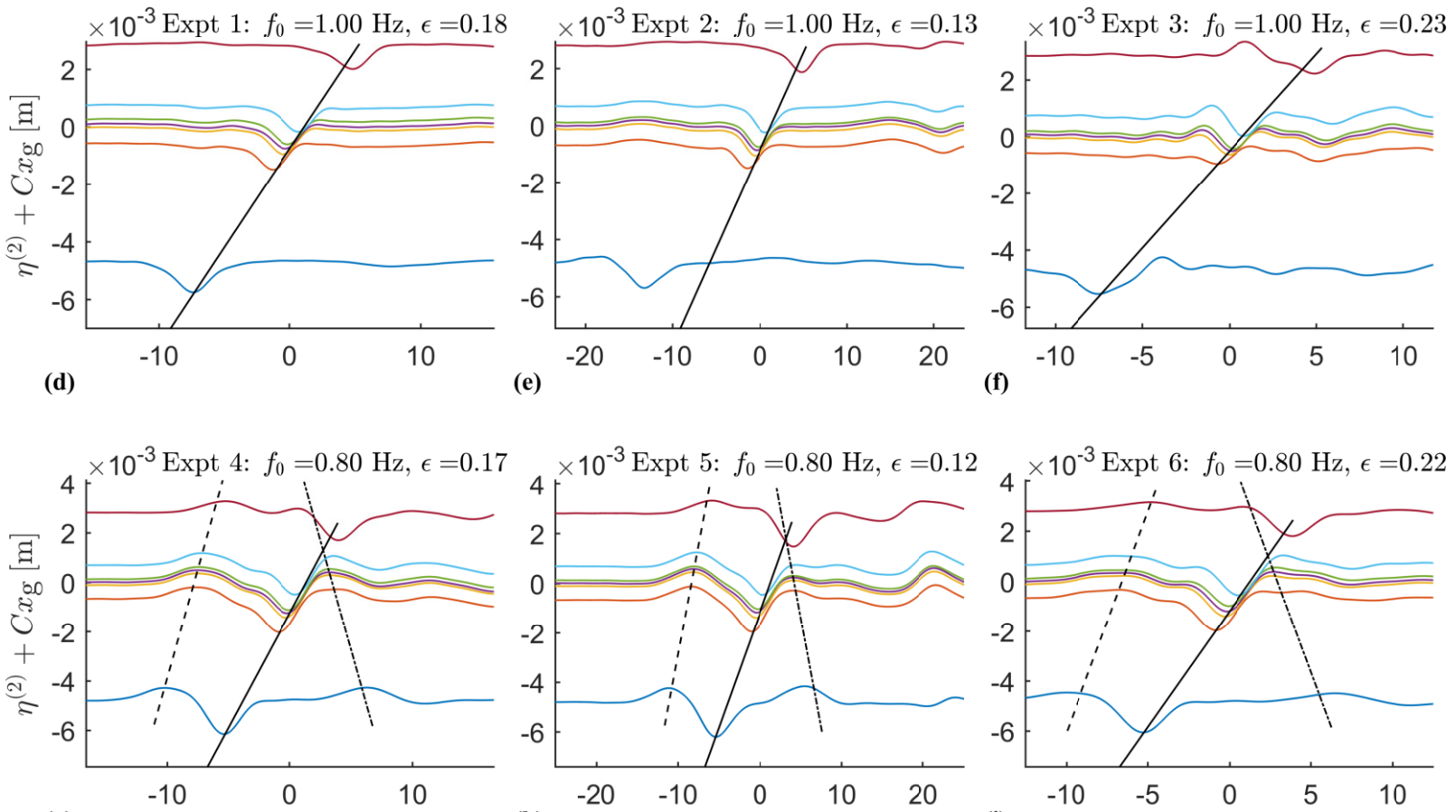

(g)

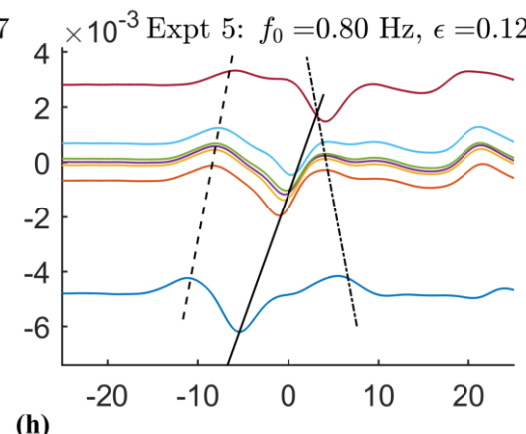

(h)
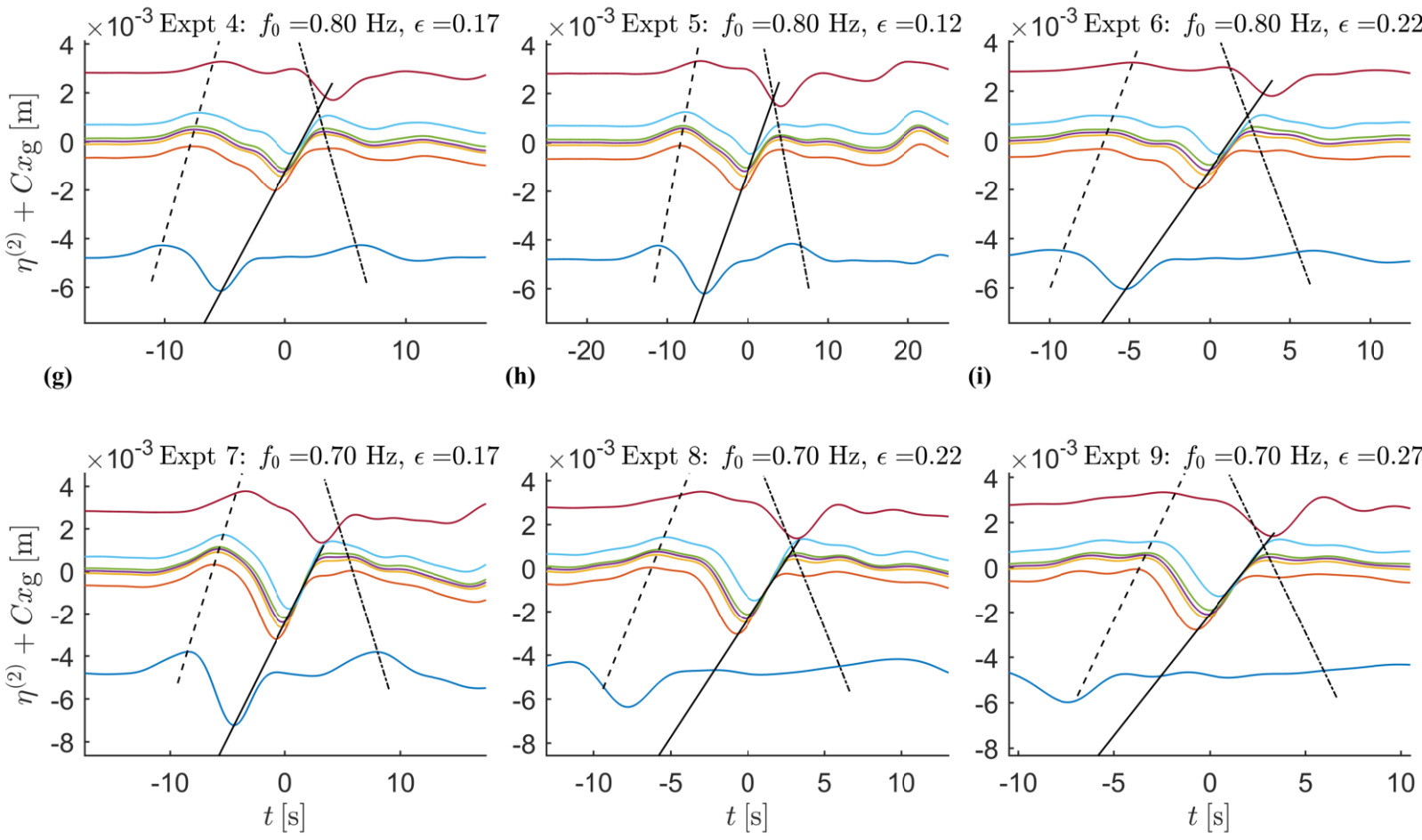

Figure 5. Time series of the sub-harmonic components of the free surface at each wave gauge (extracted from the free surface elevation using a low-pass filter at $0.5 f_{0}$ ). The $y$-axis is offset by the product of the distance of each gauge from the focus location $x_{g}$ and $C=8.0 \times 10^{-4}$. Also shown are the group speed of the wavepacket $c_{g, 0}$ with which the set-down moves (continuous black lines), the speed of the error wave $\sqrt{g d}$ (dashed black lines), and the speed of its reflection $-\sqrt{g d}$ (dash-dotted black lines).

\section{B. Particle trajectories}

Figure 7 shows typical trajectories of particles under wavepackets before removal of the background motion. The trajectories are all for experiment $1\left(f_{0}=1.0 \mathrm{~Hz}\right.$ and $\left.\epsilon=0.18\right)$. As denoted by the vertical axis, the left panel is closest to the free surface, the middle panel lower and the right panel is the deepest down in the water column. Near the free surface, there is a positive displacement because Stokes drift is dominant (shown in the left panel). Further down in the water column, the displacement reduces and changes sign; this is when the Stokes drift and the Eulerian return flow are balanced (the middle panel). Towards the bottom of the tank (right panel), there is still strong evidence of the waves in the trajectories (shown by the oscillatory motion) because these waves are finite-depth. However, the 
(a)

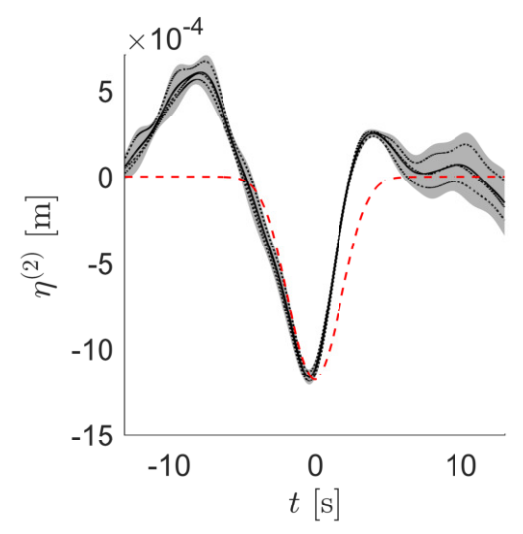

(b)

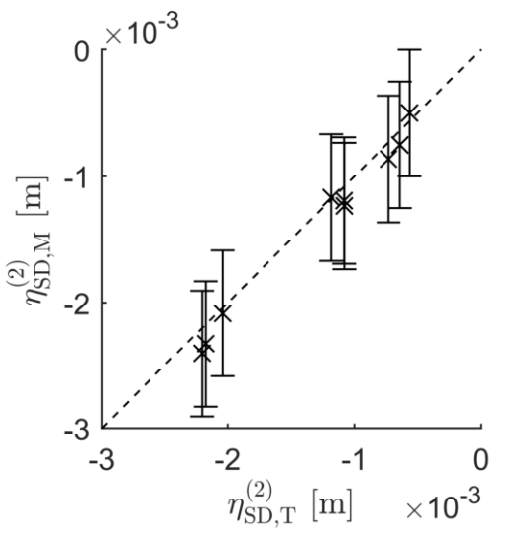

Figure 6. Quantification of the measurement error of the set-down. Panel (a) shows the sub-harmonic set-down for experiment $5\left(f_{0}=0.88 \mathrm{~Hz}, \epsilon=0.12\right)$ as a function of time: three repeats are plotted using black dotted lines, and the mean is overlaid in solid black. The grey region shows a confidence band of \pm 2 standard deviations around the mean. The theoretical set-down is plotted using a dashed red line. Panel (b) is a comparison of the magnitude of the set-down between theoretical (labeled $\eta_{\mathrm{SD}, \mathrm{T}}^{(2)}$ on the horizontal axis) and experimentally measured (labeled $\eta_{\mathrm{SD}, \mathrm{M}}^{(2)}$ on the vertical axis) magnitude of the set-down for each experiment. The error bars show \pm 2 standard deviations of the absolute calibration error.

(a)

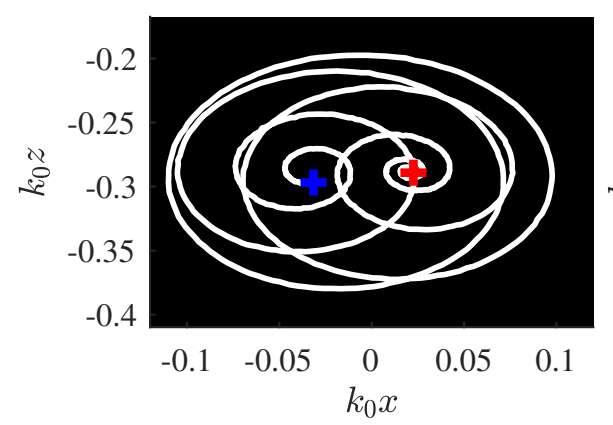

(b)

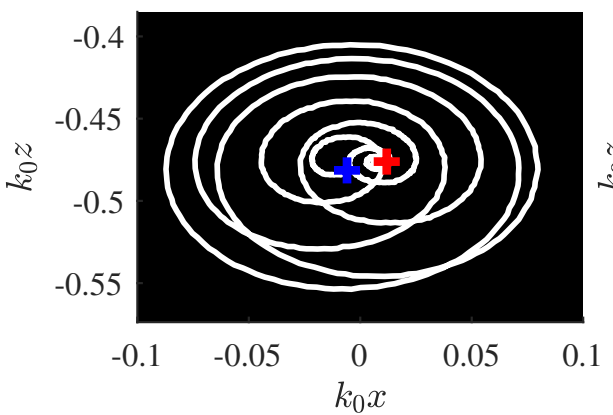

(c)

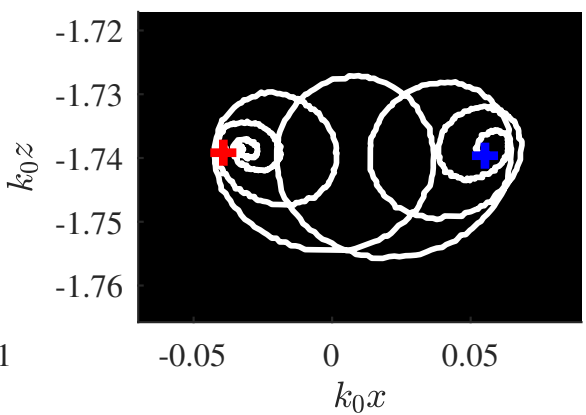

Figure 7. Experimental particle trajectories under wave packets with $f_{0}=1.00 \mathrm{~Hz}$ and $\epsilon=0.18$ at three different depths and before removal of the background motion. The left panel is closest to the surface, the central panel is close to the vertical position where there is no mean drift and the right panel is the deepest trajectory, showing the large negative mean flow. The blue and red crosses denote the position before the wavepacket has arrived and after it has passed, respectively.

Eulerian mean flow dominates the drift, and the particles move in the opposite direction (right to left) to that of wave propagation (left to right). Note that there is a small net vertical displacement in fig. 7, as the background motion has not yet been removed. The predicted and experimentally measured depth profiles after removal of the background motion are examined in the next section.

\section{Horizontal Lagrangian displacements}

Figure 8 compares the measured horizontal particle displacements with theoretical predictions based on the measured (linearized) free surface elevation. As discussed in section IV A, the displacements are calculated over the interval $t_{1}=-0.1 \sigma / c_{g, 0}$ to $t_{2}=0.1 \sigma / c_{g, 0}$, where $t=0$ is the focus time. The short time integral window $\left(t_{2}-t_{2}=0.2 \sigma c_{g, 0}\right)$ was used to avoid including displacements induced by the sub-harmonic error wave. The packet length scale $\sigma$ and 
the group velocity of the carrier $c_{g, 0}$ were estimated from fitting a Gaussian to the measured free surface signal. As expected, for an irrotational flow, Stokes drift dominates at the top of the water column resulting in positive displacements, whilst the Eulerian return flow causes opposite displacements, which dominate at depth.

Figure 8 includes two theoretical predictions: including the effect of the set-down (22) (continuous red lines) and excluding the set-down (dotted black lines). To predict the displacement using (22), we use the envelope $A_{0}$ obtained from a Hilbert transform of the linearised free surface elevation. To exclude the set-down, we replace the term $\left(1-c_{g, 0}^{2} /(g d)\right)$ in the denominator of the second term in the square bracket in (22) by 1 . It is immediately evident from fig. 8 that agreement between measurements and theoretical predictions indeed requires inclusion of the set-down. Including the effect of the set-down can be likened to a convergent-divergent duct, thus accelerating the Eulerian mean flow and resulting in a larger velocity underneath the centre of the group. The set-down grows in magnitude as the carrier wave gets shallower ( $f_{0}$ decreases) and thus forces the mean flow more significantly. As the carrier waves get shallower, $c_{g, 0}^{2} /(g d)$ approaches 1 , and thus the change in denominator creates a very large difference in the predicted return flow. This can be observed in fig. 8 by the increase in difference between the two theoretical lines as $f_{0}$ decreases (lower rows). For the experiments with the greatest depth (experiments 1-3, $k_{0} d=2.1$ ), the importance of the set-down is minimal, as is evident by the small difference between the two theoretical lines. However, for the shallower cases (experiments $4-6, k_{0} d=1.4$; experiments $7-9, k_{0} d=1.2$ ), it is evident that the prediction including the set-down is far better than without. For completeness, we note that the agreement for the shallower experiments is less good, likely, as a result of the more dominant role of imperfectly eliminated error waves in these cases.

In fig. 8, the bandwidth $\epsilon\left(\epsilon \equiv 1 /\left(k_{0} \sigma\right)\right.$ with $\sigma$ the group length) is varied for each frequency (each panel in a particular row has a different $\epsilon$ ). One can observe an increase in the magnitudes of the transport with a reduction in $\epsilon$ : the narrower the bandwidth $\epsilon$, the longer the wavepacket. Figure 9 shows scaled and non-dimensional measured horizontal displacement profiles, allowing experiments at the same relative depth $k_{0} d$ but a different group length $\sigma=1 /\left(k_{0} \epsilon\right)$ to be overlaid. The non-dimensional horizontal displacements collapse onto a single curve for the three values of $k_{0} d$ considered.

It is evident from the results that there is a mean transport observed under two-dimensional wavepackets, which can be decomposed into Stokes drift and an Eulerian return flow. Stokes drift is dominant near the surface, as evident from the positive transport near the surface, and its decay with depth matches the theory well. It is worth noting that although the displacement by Stokes drift decays with depth, the displacement by the Eulerian return flow is constant with depth. This is can be seen in eq. (22) and is consistent with the observed measured displacements.

Although the net vertical displacement by a group is zero, the measured vertical displacement underneath the centre of the group can also be compared to theory. Although the data are subject to more noise, experiments and theory also agree well here (see appendix D).

\section{CONCLUSIONS}

In this paper, we have derived from the water wave equations an expression for wave-induced Eulerian mean flow for a quasi-monochromatic or narrow-banded surface gravity wavepacket that is valid for arbitrary water depth both relative to the carrier wave and the wavepacket (or return flow) scale. In doing so, we have generalized previous results in the literature that are valid for packets and their return flows that are either very short $(d / \sigma \gg 1)$ or very long relative to the water depth $(d / \sigma \ll 1)$, referred to by us as shallow and deep return-flow, respectively. Furthermore, we have corrected van den Bremer and Taylor [44], who did take into account the effect of finite depth, but incompletely so, by ignoring the set-down. Physically, we explain the mechanism by which set-down enhances the mean flow in finite depth, namely by acting as a convergent-divergent duct.

There has been some disagreement in the literature whether a net drift should be observed in a laboratory wave flume (see the discussion in [4, 32-34]). We have clearly shown in this paper that for the relatively short groups considered, observations are in good agreement with leading-order equations of the irrotational water waves equations in two dimensions. These equations predict a net drift under wavepackets in irrotational flows. The resulting displacement is the result of both the Stokes drift and the Eulerian return flow. The Stokes drift displacement is positive (in the direction of the waves) and decays with depth on the scale of the carrier wavelength. The displacement induced by the return flow is negative and does not vary as a function of the vertical coordinate, as predicted both in the shallow return flow and in the deep return flow regimes.

Experiments were designed to extract Lagrangian particle displacements for wavepackets in finite depth $\left(k_{0} d=\right.$ $1.2-2.2$ ) in a flume without full second-order wave-generation, where the error wave is of significant amplitude and travels at a similar speed to the wavepacket. The processing technique accounted for motion from the error wave and 


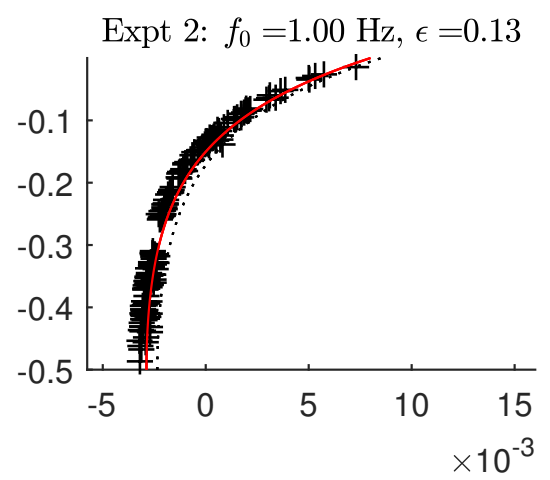

Expt 5: $f_{0}=0.80 \mathrm{~Hz}, \epsilon=0.12$

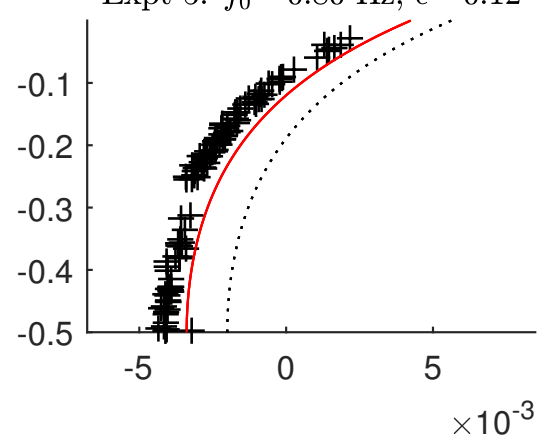

Expt 8: $f_{0}=0.69 \mathrm{~Hz}, \epsilon=0.22$

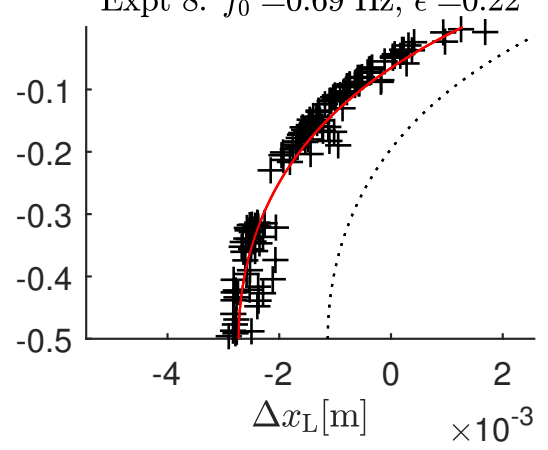

Expt 3: $f_{0}=1.00 \mathrm{~Hz}, \epsilon=0.23$

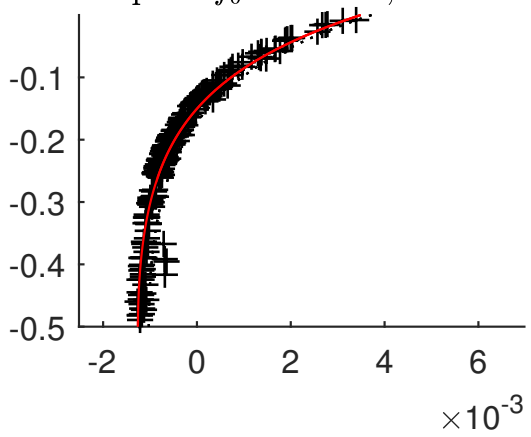

Expt 6: $f_{0}=0.80 \mathrm{~Hz}, \epsilon=0.22$

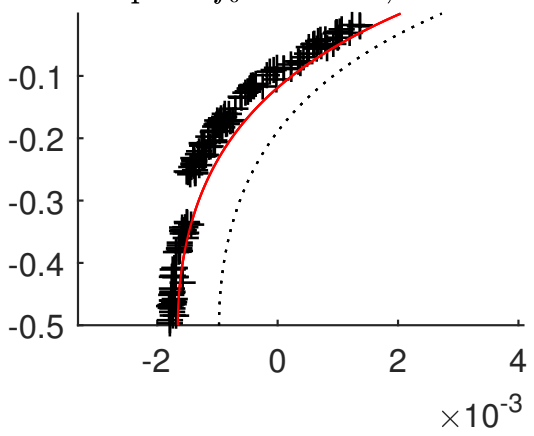

Expt 9: $f_{0}=0.70 \mathrm{~Hz}, \epsilon=0.27$

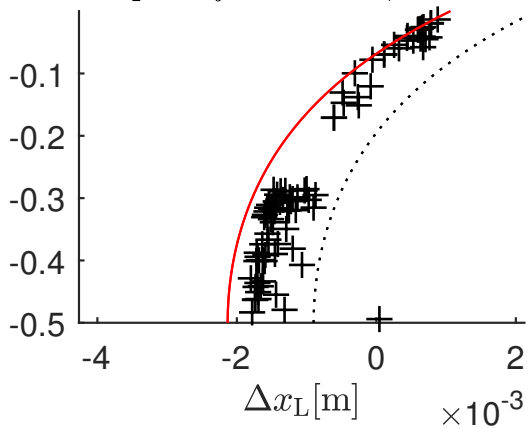

Figure 8. Profiles of horizontal Lagrangian displacement between $t_{1}=-0.1 \sigma / c_{g, 0}$ and $t_{2}=0.1 \sigma / c_{g, 0}$ (with $t=0$ the centre of the packet) as a function of depth: post-processed experimental data (black crosses), theoretical prediction from (22) (continuous red lines) and theoretical prediction ignoring the set-down from (22) (dotted black lines). The theoretical prediction ignoring the set-down is obtained by replacing the term $\left(1-c_{g, 0}^{2} /(g d)\right)$ in the denominator of the second term in the square bracket in (22) by 1 .

eliminated other background motion of the Lagrangian seeding particles. The effect of set-down in these experiments was clear, and the theoretical solution was found to match the experimental measurements far better than if the contribution from the set-down forcing was excluded. The experimental methodology presented herein enables mean flows to be measured in finite water depth despite the presence of an error wave. Although full second-order wave generation is preferable, such generation will be physically constrained in many wavemakers by the maximum length of the paddle sweep and its inability to generate all the slow bound sub-harmonic components of the signal. Careful consideration of the error wave through fitting methods on the mean flow will then be necessary, as demonstrated herein.

In shallow coastal waters, where the set-down under wavepackets is significant, the increase in mean flow found herein would significantly effect transport of pollutants (such as plastic, oil, wreckage, and sediment). Transport by the return flow is opposite to the direction of wave propagation, and, unlike the transport by the Stokes drift, does 
(a)

(b)

Expt: $1-3 k_{0} d=2.08$

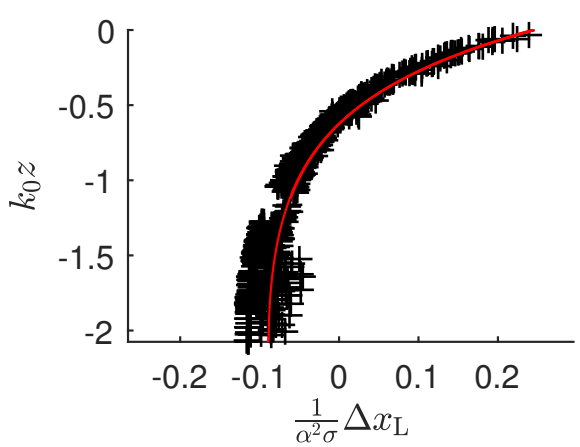

Expt: $4-6 k_{0} d=1.43$

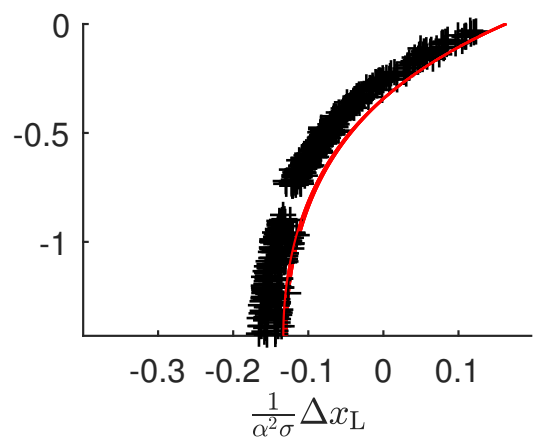

(c)

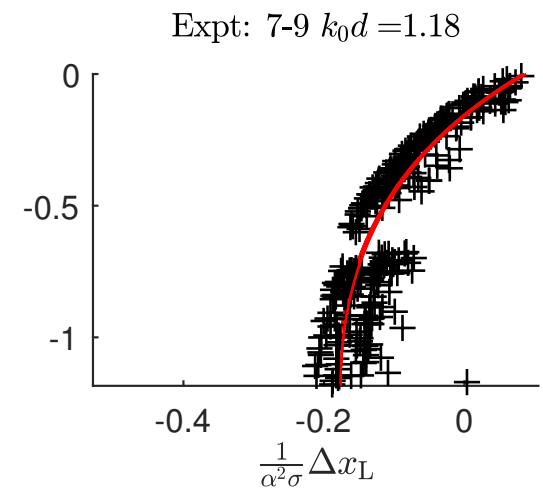

Figure 9. Scaled, non-dimensional profiles of horizontal Lagrangian displacement between $t_{1}=-0.1 \sigma / c_{g, 0}$ and $t_{2}=0.1 \sigma / c_{g, 0}$ (with $t=0$ the centre of the packet) as a function of depth: post-processed experimental data (black crosses) and theoretical predictions from (22) (continuous red lines).

not decay with depth.

\section{ACKNOWLEDGMENTS}

TSvdB was supported by a Royal Academy of Engineering Research Fellowship. The authors wish to thank Christopher Higgins and Yan Li for helpful discussions.

\section{Appendix A: Analysis of poles in wavenumber integral}

In the solution to $\phi^{(2)}(15)$ there are three poles in the integral over wavenumber. The first is at $\kappa=0$ and can be considered inconsequential because it only exists in the potential and disappears once spatially differentiated to velocity; $\kappa=0$ corresponds to a mean increase in potential (infinite wavelength), which has no physical interpretation. The second and third poles are symmetrical and stem from $\left(1-c_{g, 0}^{2} \kappa /(g \tanh \kappa d)\right)^{-1}$, which we shall define as $1 / f(\kappa)$. The poles are at $\pm \kappa_{p}$ only if the gradient $c_{g, 0}^{2} / g d<1$, which is true for all cases other than at the limit of shallow water where $c_{g, 0}^{2} /(g d)=1$. If $f(\kappa)$ is expanded around $\kappa_{p}$ in a Taylor series, it can be shown that $f^{\prime}(\kappa)$ is an odd function. Thus, when combined with the other terms in the equation for velocity, the two symmetric poles will cancel each other out upon integration.

\section{Appendix B: Comparison with existing literature}

The theory presented in section II describes a single solution for mean flow in water of arbitrary depth. Table I shows the regimes characterised by relative depths $k_{0} d$ and $d / \sigma$. In this section, the solution is shown to recover the results of these established regimes and, to the authors' knowledge, is the first to model a return flow in arbitrary depth, either of the carrier wave $\left(k_{0} d\right)$ or the return flow $(d / \sigma)$. 


\section{Shallow return flow: $k_{0} d=\mathcal{O}(1)$ and $d / \sigma \ll 1$}

The solution for $\phi^{(2)}$ in (15) does not make an assumption regarding the depth of the return flow. If the return flow is assumed to be shallow $(d / \sigma \ll 1)$, after differentiation to obtain horizontal velocity, $(15)$ simplifies to:

$$
u^{(2)}=-\frac{\omega_{0}\left(1+C_{\mathrm{FD}}\left(k_{0} d\right)\right)}{4 \pi \tanh \left(k_{0} d\right)\left(1-\frac{c_{g, 0}^{2}}{g d}\right)} \int_{-\infty}^{\infty} \kappa \widehat{\left|A_{0}\right|^{2}} \frac{\exp (\imath \kappa \tilde{x})}{\kappa d} \mathrm{~d} \kappa,
$$

and we can explicitly evaluate (16):

$$
\eta^{(2)}=-\frac{c_{g, 0}}{g} \frac{\left(1+C_{\mathrm{FD}}\left(k_{0} d\right)\right)}{2 \tanh \left(k_{0} d\right)\left(g d-c_{g, 0}^{2}\right)} g\left|A_{0}\right|^{2}-\frac{\omega_{0}^{2}\left|A_{0}\right|^{2}}{4 g \sinh ^{2}\left(k_{0} d\right)},
$$

which can be rewritten as

$$
\eta^{(2)}=-\frac{K\left(c_{g, 0}^{2} / g\right)\left|A_{0}\right|^{2}}{g d-c_{g, 0}^{2}}-\frac{\omega_{0}^{2}\left|A_{0}\right|^{2}}{4 g \sinh ^{2}\left(k_{0} d\right)}
$$

where

$$
K=\frac{\omega_{0}^{2}}{4 \sinh ^{2}\left(k_{0} d\right)}\left[\frac{\sinh \left(4 k_{0} d\right)+3 \sinh \left(2 k_{0} d\right)+2 k_{0} d}{2 k_{0} d+\sinh \left(2 k_{0} d\right)}\right] .
$$

Equation (B3) is identical to (3.17b) obtained by Longuet-Higgins and Stewart [3] under the assumption of a shallow return flow when solving for the potential ( $\phi$ in equation (3.17a) of Longuet-Higgins and Stewart). Note that LonguetHiggins and Stewart initially represent the wavepacket $A_{0}$ as a sum of difference waves, which are waves with phase equal to the difference between the that of the linear components, and do not take a multiple-scales approach.

The two terms on right-hand side of (B3) can be combined using a rearranged relation for group velocity, $k_{0} d / \sinh \left(2 k_{0} d\right)=c_{g, 0} / c_{p, 0}-1 / 2$, to give

$$
\eta^{(2)}=-\frac{g\left|A_{0}\right|^{2}}{2\left(g d-c_{g, 0}^{2}\right)}\left[\frac{2 c_{g, 0}}{c_{p, 0}}-\frac{1}{2}\right],
$$

which is the same as equation (11.4.4) obtained by Mei et al. [23] through a multiple-scales approach similar to ours.

\section{Deep return flow: $k_{0} d \gg 1$ and $d / \sigma \gg 1$}

Using their non-dimensional notation, our forcing equations (5) and (13) can be rewritten as (2.20-2.21) in Dysthe [24]. As noted by Dysthe [24], the $\epsilon^{2} \partial_{X X} \phi^{(2)}$ term in (13) can be ignored in deep water, and an explicit solution for the return flow velocity can be found [25],

$$
u^{(2)}=-\frac{\omega_{0}}{4 \pi} \int_{-\infty}^{\infty} \kappa \widehat{\left|A_{0}\right|^{2}} \exp (\kappa z) \exp (\imath \kappa \tilde{x}) \mathrm{d} \kappa .
$$

\section{Appendix C: Trajectory processing}

The raw motion of the seeding particles also included background motion and motion due to the error wave, which was significantly larger in the shallower experiments. This motion had to be eliminated, and a suitable interval to calculate displacements used to achieve good agreement of the experimentally measured displacements with the theory. The background motion of particles is much slower than the Stokes drift and the Eulerian return flow beneath the group. We eliminated background motion using a suitable curve fit (selected from four possible fits) to the motion of the particles over specified time interval(s) before and/or after the arrival of the focused wavepacket and then subtract the fit from the original $x(t)$ and $z(t)$ trajectories. The post-processing algorithm first identified the focus time $t_{f}$ within the record as the time of the maximum horizontal velocity, and calculated the group velocity $c_{g, 0}$, and hence the group period $T_{g}$, by solving the linear dispersion equation based on the modulation of linear surface signal, $A_{0}$. The group period formed the basis of the 'fit windows' used by the different curve fit options: 
a) Linear fit to $x$-displacement prior to the wavepacket arrival $\left(t_{f}-6 T_{g}\right.$ to $\left.t_{f}-3 T_{g}\right)$, quadratic fit to $z$-displacement before and after the wavepacket arrival $\left(t_{f} \pm 6 T_{g}\right.$ to $\left.t_{f} \pm 3 T_{g}\right)$.

b) Linear fit to $x$-displacement prior to the wavepacket arrival $\left(t_{f}-12 T_{g}\right.$ to $\left.t_{f}-9 T_{g}\right)$, quadratic fit to $z$-displacement before and after the wavepacket arrival $\left(t_{f} \pm 9 T_{g}\right.$ to $\left.t_{f} \pm 3 T_{g}\right)$.

c) Linear fit to $x$-displacement prior to the wavepacket arrival $\left(t_{f}-12 T_{g}\right.$ to $\left.t_{f}-3 T_{g}\right)$, quadratic fit to $z$-displacement before and after the wavepacket arrival $\left(t_{f} \pm 12 T_{g}\right.$ to $\left.t_{f} \pm 3 T_{g}\right)$.

d) Linear fit to $x$-displacement prior to the wavepacket arrival $\left(t_{f}-9 T_{g}\right.$ to $\left.t_{f}-3 T_{g}\right)$, quadratic fit to $z$-displacement before and after the wavepacket arrival $\left(t_{f} \pm 9 T_{g}\right.$ to $\left.t_{f} \pm 3 T_{g}\right)$.

Once the fits to the raw motions had been carried out, the frequency spectrum of the motion was examined (the motion was first mirrored to create a periodic signal). Using the knowledge that for narrow-banded wavepackets the sub-harmonic and periodic motion should be well separated (this is not the case for background motion if this is not periodic), the fitting method that led to the frequency spectrum which was most separated was chosen for each trajectory.

The remaining signal was then filtered with a low-pass filter to eliminate the periodic motion; this allowed a accumulated displacement over any time to be compared with theory. For the horizontal motion, an interval of $\pm 0.1 \sigma / c_{g, 0}$ was used to avoid displacement by the error wave. The vertical displacements were measured over half of the wavepacket before the time of focus $\left(t_{1}=-3 T_{g}\right.$ and $\left.t_{2}=0\right)$. If only a small window before focus was used for the vertical displacement, the measurements became very small and dominated by noise.

\section{Appendix D: Vertical Lagrangian displacements}

Figure 10 compares the measured vertical Lagrangian displacement profiles with theoretical predictions based on the measured (linearized) free surface elevation from (24). The time integral in (24) has been taken from before the group arrives $\left(t_{1}=-3 \sigma / c_{g, 0}\right)$ to focus time $\left(t_{2}=0\right)$. The agreement between measured and predicted vertical displacements appears less satisfactory than for horizontal displacements because the values are an order of magnitude smaller, leading to larger relative errors. Even so, measurements capture predictions in terms of their shape and magnitude, including zero vertical displacements at the bottom, negative displacements at mid depth due to the return flow and positive displacements near the free surface due to Stokes drift. Measurements and theory agree better in deeper water (experiment 1-3 compared to experiments 4-9). This is most likely because the effect of the error wave is larger in the shallower cases. Figure 11 shows scaled and non-dimensional measured vertical displacement profiles, allowing experiments at the same relative depth $k_{0} d$ but a different group length $\sigma=1 /\left(k_{0} \epsilon\right)$ to be overlaid. The measured vertical displacements again show far more scatter than their horizontal counterparts. The increased magnitude of the error wave at low $k_{0} d$ is evident in the additional scatter in the experiments at shallower relative depth.

[1] G. G. Stokes. On the theory of oscillatory waves. Camb. Trans., 8:441, 1847.

[2] M. S. Longuet-Higgins. The statistical analysis of a random, moving surface. Phil. Trans. R. Soc. Lond. A, 249(966): 321-387, 1957.

[3] M. S. Longuet-Higgins and R.W. Stewart. Radiation stress and mass transport in gravity waves, with application to 'surf beats'. J. Fluid Mech., 13:481-504, 1962.

[4] T. S.van den Bremer and Ø. Breivik. Stokes drift. Phil. Trans. R. Soc. A, 376(2111):20170104, 2018.

[5] M. Cole, P. Lindeque, C. Halsband, and T. S. Galloway. Microplastics as contaminants in the marine environment: a review. Mar. Pollut. Bull, 62(12):2588-2597, 2011.

[6] A. Cózar, F. Echevarrí, J. I. González-Gordillo, X. Irigoien, B. Úbeda, S. Hernández-León, Á. T. Palma, S. Navarro, J. García de Lomas, A. Ruiz, et al. Plastic debris in the open ocean. P. Natl. Acad. Sci., 111(28):10239-10244, 2014.

[7] A. Isobe, K. Kubo, Y. Tamura, E. Nakashima, N. Fujii, et al. Selective transport of microplastics and mesoplastics by drifting in coastal waters. Mar. Pollut. Bull, 89(1):324-330, 2014.

[8] P. Sherman and E. Van Sebille. Modeling marine surface microplastic transport to assess optimal removal locations. Environ. Res. Lett., 11(1):014006, 2016.

[9] J. A. Trinanes, M. J. Olascoaga, G. J. Goni, N. A. Maximenko, D. A. Griffin, and J. Hafner. Analysis of flight mh370 potential debris trajectories using ocean observations and numerical model results. J. Operat. Oceanogr., 9(2):126-138, 2016. 


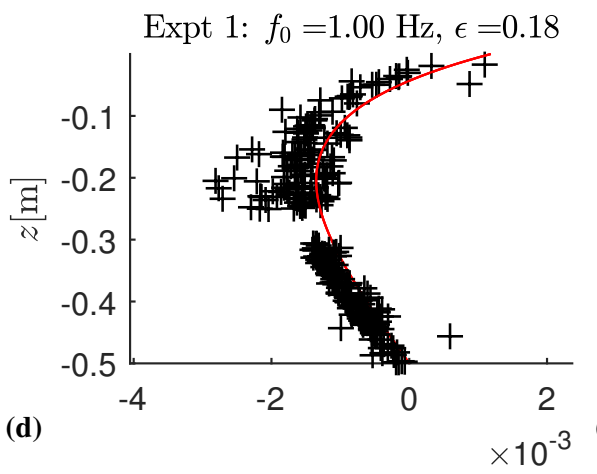

(e)
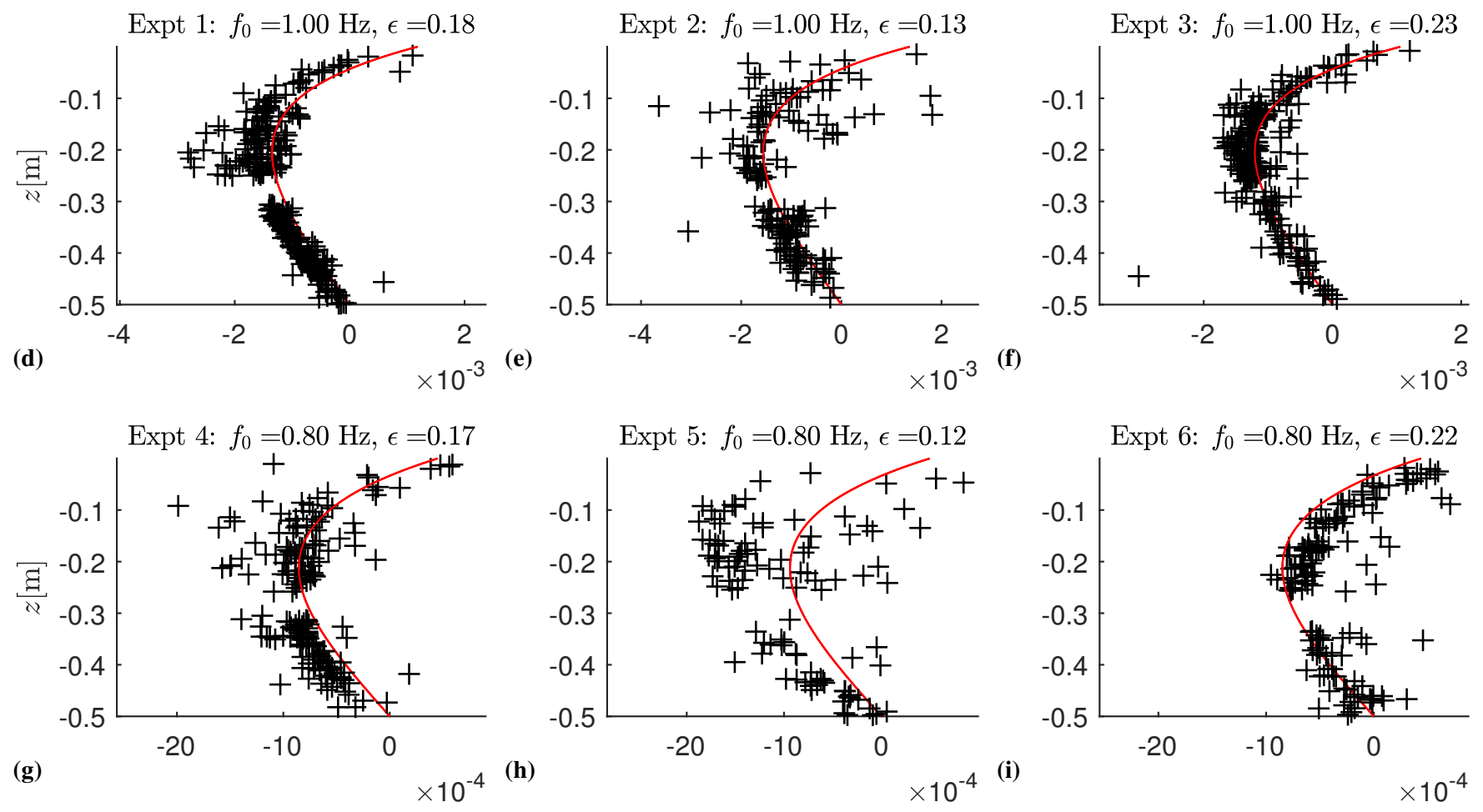

Expt 7: $f_{0}=0.69 \mathrm{~Hz}, \epsilon=0.17$
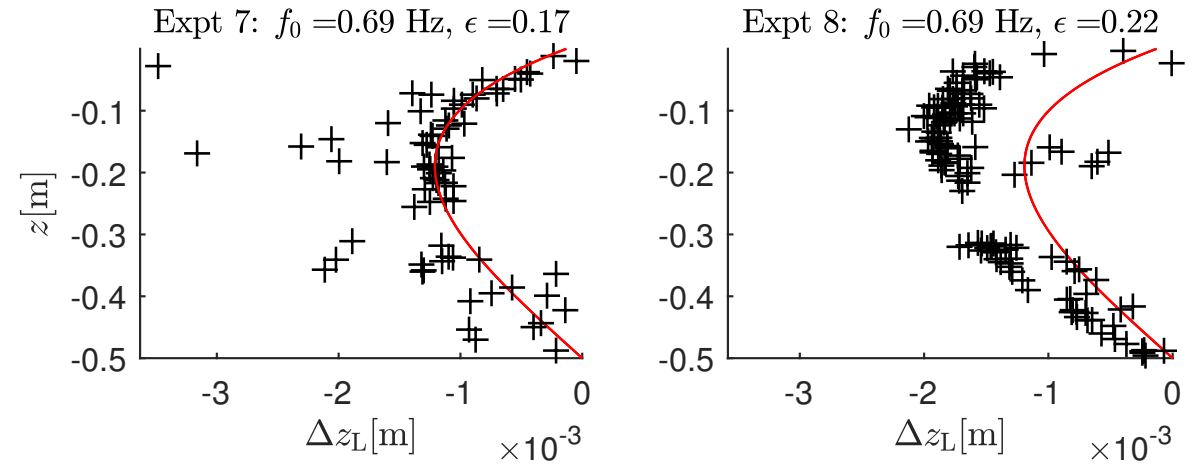

Expt 9: $f_{0}=0.70 \mathrm{~Hz}, \epsilon=0.27$

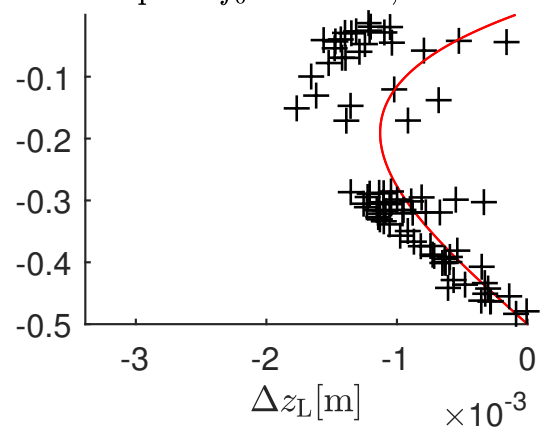

Figure 10. Profiles of vertical Lagrangian displacement between $t_{1}=-3 \sigma / c_{g, 0}$ and $t_{2}=0$ (corresponding to the centre of the packet $t=0$ ) as a function of depth: post-processed experimental data (black crosses) and theoretical predictions from (24) (continuous red lines).

[10] K. H. Christensen and E. Terrile. Drift and deformation of oil slicks due to surface waves. J. Fluid Mech., 620:313-332, 2009.

[11] M. Drivdal, G. Broström, and K. H. Christensen. Wave-induced mixing and transport of buoyant particles: application to the statfjord a oil spill. Ocean Sci., 10(6):977-991, 2014.

[12] C. E. Jones, K.-F.Dagestad, Ø. Breivik, B. Holt, J. Röhrs, H. Christensen, M. Espeseth Kai, C. Brekke, and S. Skrunes. Measurement and modeling of oil slick transport. JGR: Oceans, 121(10):7759-7775, 2016.

[13] R. Deigaard et al. Mechanics of coastal sediment transport, volume 3. World Scientific Publishing Company, 1992.

[14] P. Nielsen. Coastal bottom boundary layers and sediment transport, volume 4. World Scientific Publishing Company, 1992.

[15] M. E. McIntyre. On the 'wave momentum' myth. J. Fluid Mech., 106:331-347, 1981.

[16] J. Orszaghova, P. H. Taylor, A. G. L. Borthwick, and A. C. Raby. Importance of second-order wave generation for focused wave group run-up and overtopping. Coast. Eng., 94:63-79, 2014.

[17] C. N. Whittaker, C. J. Fitzgerald, A. C. Raby, P. H. Taylor, J. Orszaghova, and A. G. L. Borthwick. Optimisation of focused wave group runup on a plane beach. Coast. Eng., 121:44 - 55, 2017.

[18] P. Nielsen and T. Baldock. И-shaped surf beat understood in terms of transient forced long waves. Coast. Eng., 57, 01 2010. 


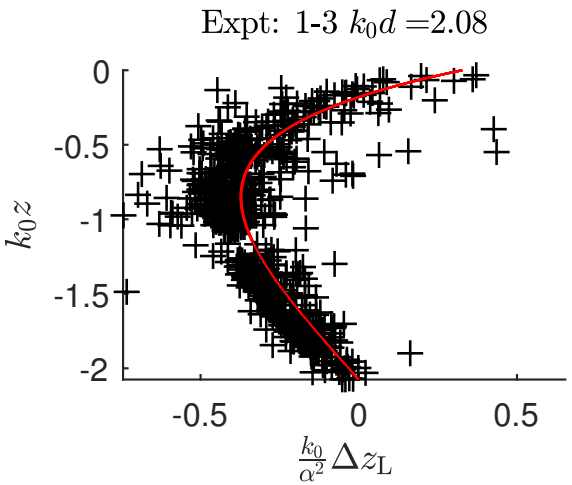

Expt: $7-9 k_{0} d=1.18$

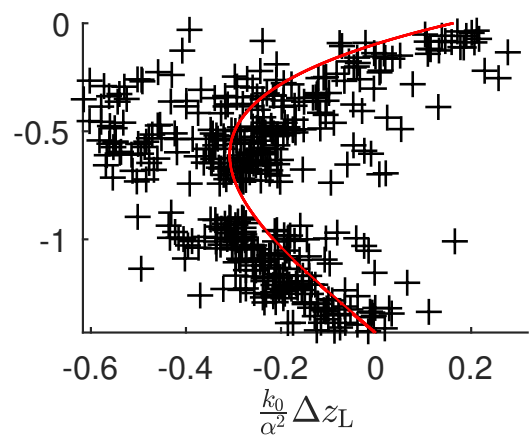

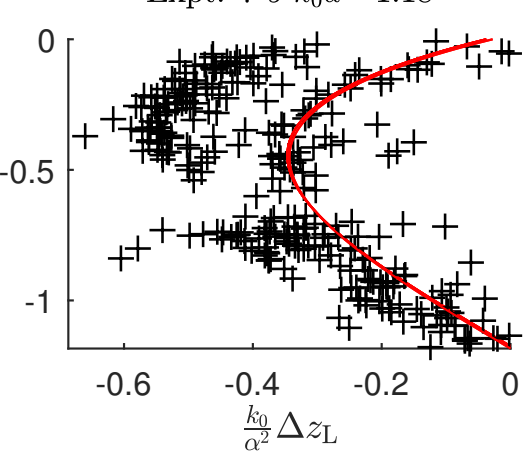

Figure 11. Scaled, non-dimensional profiles of vertical Lagrangian displacement between $t_{1}=-3 \sigma / c_{g, 0}$ and $t_{2}=0$ (corresponding to the centre of the packet $t=0$ ) as a function of depth: post-processed experimental data (black crosses) and theoretical predictions from (24) (continuous red lines).

[19] H. A. Schäffer. Second-order wavemaker theory for irregular waves. Ocean Eng., 23(1):47-88, 1996.

[20] P. J. Van Leeuwen and G. Klopman. A new method for the generation of second-order random waves. Ocean Eng., 23(2): 167-192, 1996.

[21] A. Davey and K. Stewartson. On three-dimensional packets of surface waves. Phil. Trans. R. Soc. Lond. A, 338:101-110, 1974.

[22] U. Brinch-Nielsen and I. G. Jonsson. Fourth order evolution equations and stability analysis for Stokes waves on arbitrary water depth. Wave Motion, 8:455-472, 1986.

[23] C. C. Mei, M. Stiassnie, and D. K.-P. Yue. Theory and Applications of Ocean Surface Waves: part 2: Nonlinear Aspects. World Scientific, 1989.

[24] K. B. Dysthe. Note on a modification to the nonlinear Schrödinger equation for application to deep water waves. In sPhil. Trans. R. Soc. Lond. A, volume 369, pages 105-114, 1979.

[25] T. S. van den Bremer and P. H. Taylor. Lagrangian transport for two-dimensional deep-water surface gravity wave groups. Phil. Trans. R. Soc. Lond. A, 472:20160159, 2016.

[26] J. F. Dalzell. A note on finite depth second-order wave-wave interactions. Appl. Ocean. Res., 21(3):105-111, 1999.

[27] K. Hasselmann. On the non-linear energy transfer in a gravity-wave spectrum part 1. General theory. J. Fluid Mech., 12: 481-500, 1962.

[28] M. Okihiro, R.T. Guza, and R.J. Seymour. Bound infra-gravity waves. J. Geophys. Res., 97:453-469, 1992.

[29] J. N. Sharma and R. G. Dean. Second-order directional seas and associated wave forces. Soc. Petrol. Eng. J., 21:129-140, 1981.

[30] G. Z. Forristall. Wave crest distributions: Observations and second-order theory. J. Phys. Oceanogr., 30:1931-1943, 2000.

[31] L. Pellet, P. Christodoulides, S. Donne, C.J. Bean, and F. Dias. Pressure induced by the interaction of water waves with nearly equal frequencies and nearly opposite directions. Theoretical and Applied Mechanics Letters, 2017.

[32] S. G. Monismith, E.A. Cowen, H. M. Nepf, J. Magnaudet, and L. Thais. Laboratory observations of mean flows under surface gravity waves. J. Fluid Mech., 573:131-147, 2007.

[33] J. Grue and J. Kolaas. Experimental particle paths and drift velocity in steep waves at finite water depth. J. Fluid Mech., 810:R1, 2017.

[34] L. Deike, N. Pizzo, and W. K. Melville. Lagrangian transport by breaking surface waves. J. Fluid Mech., 829:364-391, 2017.

[35] M. Umeyama. Eulerian-Lagrangian analysis for particle velocities and trajectories in a pure wave motion using particle image velocimetry. Philos. T. Roy. Soc. A, 370(1964):1687-1702, 2012.

[36] M. Paprota, W. Sulisz, and A. Reda. Experimental study of wave-induced mass transport. J. Hydraul. Res., 54(4):423-434, 2016.

[37] M.S. Longuet-Higgins. Mass transport in water waves. Phil. Trans. R. Soc. Lond. A, 245:535-581, 1953.

[38] R. A. Bagnold. Sand movement by waves: some small-scale experiments with sand of very low density. J. Inst. Civil Engng., 27(4):447-469, 1947.

[39] C. Swan. Convection within an experimental wave flume. J. Hydraul. Res., 28:273-282, 1990.

[40] J. Groeneweg and G. Klopman. Changes of the mean velocity profiles in the combined wave-current motion described in a GLM formulation. J. Fluid Mech., 370:271-269, 1998.

[41] W. K. Melville and R. Rapp. The surface velocity field in steep and breaking waves. J. Fluid Mech., 189:1-22, 1988.

[42] N. E. Pizzo. Surfing surface gravity waves. J. Fluid Mech., 823:316-328, 2017.

[43] T. S. van den Bremer, C. Whittaker, R. Calvert, A. Raby, and P. H. Taylor. Experimental study of particle trajectories below deep-water surface gravity wave groups. J. Fluid Mech., in press. 
[44] T. S. van den Bremer and P. H. Taylor. Estimates of Lagrangian transport by surface gravity wave groups: The effects of finite depth and directionality. J. Geophys. Res. Oceans, 120(4):2701-2722, 2015.

[45] M.S. Longuet-Higgins and R.W. Stewart. Radiation stresses in water waves; a physical discussion, with applications. Deep-Sea Res., 2:529-562, 1964.

[46] M. L. McAllister, T. A. A. Adcock, P. H. Taylor, and T. S. van den Bremer. The set-down and set-up of directionally spread and crossing surface gravity wave groups. J. Fluid Mech., 835:131-169, 2018.

[47] T. S. van den Bremer, H. Yassin, and B. R. Sutherland. Lagrangian transport by vertically confined internal gravity wavepackets. J. Fluid Mech., 864:348-380, 2019.

[48] D. H. Peregrine. Water waves, nonlinear Schrödinger equations and their solutions. The Journal of the Australian Mathematical Society. Series B. Applied Mathematics, 25:16-43, 1983.

[49] V. Sriram, T. Schlurmann, and S. Schimmels. Focused wave evolution using linear and second order wavemaker theory. Appl. Ocean Res., 53:279-296, 2015.

[50] R. Nokes. Streams 2.02: System theory and design. University of Canterbury, 2014. 